\title{
Two-Field Integrable Evolutionary Systems of the Third Order and Their Differential Substitutions
}

\author{
Anatoly G. MESHKOV and Maxim Ju. BALAKHNEV \\ Orel State Technical University, Orel, Russia \\ E-mail:a_meshkov@orel.ru,maxibal@yandex.ru
}

Received October 04, 2007, in final form January 17, 2008; Published online February 09, 2008

Original article is available at http://www.emis.de/journals/SIGMA/2008/018/

\begin{abstract}
A list of forty third-order exactly integrable two-field evolutionary systems is presented. Differential substitutions connecting various systems from the list are found. It is proved that all the systems can be obtained from only two of them. Examples of zero curvature representations with $4 \times 4$ matrices are presented.
\end{abstract}

Key words: integrability; symmetry; conservation law; differential substitutions; zero curvature representation

2000 Mathematics Subject Classification: 37K10; 35Q53; 37K20

\section{Introduction}

We use the term "integrability" in the meaning that a system or equation under consideration possesses a Lax representation or a zero curvature representation. Such systems can be solved by the inverse spectral transform method (IST) [1,2]. Exactly integrable evolution systems are of interest both for mathematics and applications. In particular, systems of the following form

$$
u_{t}=u_{x x x}+F\left(u, v, u_{x}, v_{x}, u_{x x}, v_{x x}\right), \quad v_{t}=a v_{x x x}+G\left(u, v, u_{x}, v_{x}, u_{x x}, v_{x x}\right),
$$

where $a$ is a constant, excite great interest since about 1980. The paper [3] is devoted to construction of systems of the form (1.1) among others. Nine integrable systems of the form (1.1) and their Lax representations have been obtained in the paper. In particular, it contains a complete list of three integrable systems (1.1) satisfying the conditions $a(a-1) \neq 0$ and $\operatorname{ord}(F, G)<2$. Here ord $=$ order, ord $f<n$ means that $f$ does not depend on $u_{n}, v_{n}, u_{n+1}, v_{n+1}, \ldots$ Here and in what follows, the notations $u_{n}=\partial^{n} u / \partial x^{n}, v_{n}=\partial^{n} v / \partial x^{n}$ are used.

Two of the three mentioned systems can be written in the following form

$$
\begin{array}{ll}
u_{t}=u_{3}+v u_{1}, & v_{t}=-\frac{1}{2} v_{3}+u u_{1}-v v_{1}, \\
u_{t}=u_{3}+v u_{1}, & v_{t}=-\frac{1}{2} v_{3}-v v_{1}+u_{1}
\end{array}
$$

and the third system is presented below (see (3.46a)). System (1.2) was found independently in [4] and the soliton solutions were constructed there. This system is called as the DrinfeldSokolov-Hirota-Satsuma system.

This paper contains two results: (i) a list of integrable systems of the form (1.1) with smooth functions $F, G$ and $a=-1 / 2$; (ii) differential substitutions that allow to connect any equation from the list with (1.2) or (1.3).

There are many articles dealing with integrable systems, but some of them (see, e.g., [5, 6, 7]) consider multi-component systems. Other papers (see, e.g., $[8,9,10,11,12]$ ) contain twocomponent systems reducible to a triangular form. The triangular form is briefly considered below. There was possibly only one serious attempt [13] to classify integrable systems of the form (1.1) using the Painlevé test. Unfortunately, fifteen systems presented in [13] contain a large 
number of constants some of which can be removed by scaling and linear transformations. Note that there are two-field integrable evolutionary systems $\boldsymbol{u}_{t}=A \boldsymbol{u}_{3}+\boldsymbol{H}\left(\boldsymbol{u}, \boldsymbol{u}_{1}, \boldsymbol{u}_{2}\right)$ with a nondiagonal main matrix $A$. For example, an integrable evolutionary system with the Jordan main matrix is found in [14].

Moreover, about 50 two-field integrable systems of the form (1.1) with $a=1$ can be extracted from papers $[15,16,17,18,19]$ that deal with vector evolutionary equations.

Partial solutions of the classification problem for $a=0$ and ord $G \leqslant 1$ have been obtained in [20], and in [21] for divergent systems with $a \neq 1$. A complete list of integrable systems of the form (1.1) does not exist today because the problem is too cumbersome and the set of integrable systems is very large.

Our tool is the symmetry method presented in many papers. We shall point out pioneer or review papers only. In [22] the notions of formal symmetry and canonical conserved density for a scalar evolution equation are introduced. These tools were applied to classification of the KdVtype equations in [23]. A complete theory of formal symmetries and formal conservation laws for scalar equations has been presented in [24]. A generalized theory was developed for evolutionary systems in [25]. Review paper [26] contains both general theorems of the symmetry method and classification results on integrable equations: the third and fifth order scalar equations, Schrödinger-type systems, Burgers-type equations and systems. Review paper [27] is devoted to higher symmetries, exact integrability and related problems. Peculiarities of systems (1.1) have been discussed in [21]. For the sake of completeness, the main points of the symmetry method and some results necessary for understanding of this paper are considered in the Sections 2-4.

Briefly speaking, the symmetry method deals with the so-called canonical conservation laws

$$
D_{t} \rho_{n}=D_{x} \theta_{n}, \quad D_{t} \tilde{\rho}_{n}=D_{x} \tilde{\theta}_{n}, \quad n=0,1,2, \ldots,
$$

where $D_{t}$ is the evolutionary derivative and $D_{x}$ is the total derivative with respect to $x$. In particular, $\rho_{0}=-F_{u_{2}} / 3, \tilde{\rho}_{0}=-G_{v_{2}} /(3 a)$. The recursion relations for the canonical conserved densities $\rho_{n}$ and $\tilde{\rho}_{n}$ are presented in Section 2. All canonical conserved densities are expressed in terms of functions $F$ and $G$. That is why equations (1.4) impose great restrictions on the forms of $F$ and $G$. Equations (1.4) are solvable in the jet space iff

$$
E_{\alpha} D_{t} \rho_{n}=0, \quad E_{\alpha} D_{t} \tilde{\rho}_{n}=0, \quad \alpha=1,2, \quad n=0,1,2, \ldots
$$

(see [28], for example). Here

$$
E_{\alpha} \equiv \frac{\delta}{\delta u^{\alpha}}=\sum_{n=0}^{\infty}\left(-D_{x}\right)^{n} \frac{\partial}{\partial u_{n}^{\alpha}}, \quad\left(u^{1}=u, u^{2}=v\right)
$$

is the Euler operator.

Conservation law with $\rho=D_{x} \chi, \theta=D_{t} \chi$ is called trivial and the conserved density of the form $\rho=D_{x} \chi$ is called trivial too. This can be written in the form $\rho \in \operatorname{Im} D_{x}$, where $\operatorname{Im}=\operatorname{Image}$. If $\rho_{1}-\rho_{2} \in \operatorname{Im} D_{x}$, then the densities $\rho_{1}$ and $\rho_{2}$ are said to be equivalent.

There are a lot of systems in the following form

$$
u_{t}=u_{x x x}+F\left(u, u_{x}, u_{x x}\right), \quad v_{t}=a v_{x x x}+G\left(u, v, u_{x}, v_{x}, u_{x x}, v_{x x}\right),
$$

satisfying the integrability conditions (1.4). Such systems containing one independent equation are said to be triangular. It follows from the integrability conditions that the equation for $u$ must be one of the known integrable equations ( $\mathrm{KdV}, \mathrm{mKdV}$ etc). The second equation is usually linear with respect to $v, v_{x}$ and $v_{x x}$. Triangular systems do not possess any Lax representations and are not integrable in this sense. Therefore triangular systems and those reducible to the triangular form have been omitted as trivial. 
The system of two independent equations

$$
u_{t}=u_{x x x}+F\left(u, u_{x}, u_{x x}\right), \quad v_{t}=a v_{x x x}+G\left(v, v_{x}, v_{x x}\right),
$$

will be called disintegrated. It is obvious that the disintegrated form is a partial case of the triangular form. Therefore the disintegrated systems and those reducible to them have been omitted.

System (1.1) will be called reducible if it is triangular or can be reduced to triangular or disintegrated form. Otherwise, the system will be called irreducible.

Our computations show that for irreducible integrable systems (1.1) parameter $a$ must belong to the following set:

$$
A=\left\{0,-2,-\frac{1}{2},-\frac{7}{2}+\frac{3}{2} \sqrt{5},-\frac{7}{2}-\frac{3}{2} \sqrt{5}\right\} .
$$

These values were found first in [3] and were repeated in [29]. The value of $a$ is always defined at the end of computations when functions $F$ and $G$ have been found and only some coefficients are to be specified from the fifth or seventh integrability conditions (see example in Section 3.1). This means that it is enough to verify conditions (1.5) for $n=0, \ldots, 7$ and $\alpha=1,2$ to obtain $F, G$ and $a$. But for absolute certainty we have verified conditions (1.5) for $n=8,9$ and $\alpha=1,2$ for each system.

The presented set $A$ consists of zero and two pairs $\left(a, a^{-1}\right)$. The transformation $t^{\prime}=a t$, $u^{\prime}=v, v^{\prime}=u$ changes the parameter $a \neq 0$ in (1.1) into $a^{-1}$. That is why one ought to consider the values $\left\{0,-\frac{1}{2},-\frac{7}{2}+\frac{3}{2} \sqrt{5}\right\}$ of the parameter $a$. Integrable systems with $a=0$ were mentioned above, see also [30]. This paper is devoted to investigation of the case $a=-1 / 2$ only. The case $a=-\frac{7}{2}+\frac{3}{2} \sqrt{5}$ will be presented in another paper.

Section 2 contains recursion formulas for the canonical densities. The origin of the notion, some examples and a preliminary classification are considered.

A list of forty integrable systems and an example of computations are presented in Section 3.

Section 4 contains differential substitutions that connect all systems from the list. The method of computations and an example are considered. It is shown that all systems from the list presented in Section 3 can be obtained from (1.2) and (1.3) by differential substitutions.

Section 5 is devoted to zero curvature representations. The zero curvature representations for systems (1.2) and (1.3) are obtained from the Drinfeld-Sokolov $L$-operators. A method of obtaining zero curvature representations for other systems is demonstrated.

\section{Canonical densities}

One of the main objects of the symmetry approach to classification of integrable equations is the infinite set of the canonical conserved densities. Let us demonstrate how canonical conserved densities can be obtained the from the asymptotic expansions for eigenfunctions of the Lax operators. The simplest Lax equations concerned with the KdV equation

$$
u_{t}=6 u u_{x}-u_{x x x}
$$

take the following form

$$
\begin{aligned}
& \psi_{x x}-u \psi-\mu^{2} \psi=0, \\
& \psi_{t}=-4 \psi_{x x x}+6 u \psi_{x}+3 u_{x} \psi+4 \mu^{3} \psi .
\end{aligned}
$$

Here $u$ is a solution of the $\mathrm{KdV}$ equation and $\mu$ is a parameter. The standard substitution

$$
\psi=\exp \left(\int \rho d x\right)
$$


reduces equations (2.1) and (2.2) to the Riccati form

$$
\begin{aligned}
& \rho_{x}+\rho^{2}-u-\mu^{2}=0, \\
& \partial_{t} \int \rho d x=-4\left(\partial_{x}+\rho\right)^{2} \rho+6 u \rho+3 u_{x}+4 \mu^{3} .
\end{aligned}
$$

Differentiating temporal equation (2.4) with respect to $x$ one can rewrite it, using (2.3), as the continuity equation:

$$
\rho_{t}=\partial_{x}\left[\left(2 u-4 \mu^{2}\right) \rho-u_{x}\right]
$$

To construct an asymptotic expansion one ought to set

$$
\rho=\mu+\sum_{n=0}^{\infty} \rho_{n}(-2 \mu)^{-n}
$$

Then equation (2.3) results in the following well known recursion formula [2]

$$
\rho_{n+1}=D_{x} \rho_{n}+\sum_{i=1}^{n-1} \rho_{i} \rho_{n-i}, \quad n=1,2, \ldots, \quad \rho_{0}=0, \quad \rho_{1}=-u,
$$

and (2.5) results in infinite sequence of conservation laws:

$$
D_{t} \rho_{n}=D_{x}\left(2 u \rho_{n}-\rho_{n+2}\right), \quad n>0 .
$$

We change here $\partial_{t} \rightarrow D_{t}$ and $\partial_{x} \rightarrow D_{x}$ because $u$ is a solution of the KdV equation. The obtained conservation laws are canonical. It is easy to obtain several first canonical densities:

$$
\rho_{2}=-u_{1}, \quad \rho_{3}=u^{2}-u_{2}, \quad \rho_{4}=D_{x}\left(2 u^{2}-u_{2}\right), \quad \ldots
$$

It is shown in [2] that all even canonical densities are trivial. Note that if one chooses another asymptotic expansion, for example, in powers of $\mu^{-1}$ instead of (2.6), then another set of canonical densities is obtained, which is equivalent to the previous set.

The canonical densities that follow from (2.7) can also be obtained by using the temporal equation (2.4) only. Indeed, setting $\partial_{t} \int \rho d x=\theta$ one obtains from (2.4)

$$
-4\left(\partial_{x}+\rho\right)^{2} \rho+6 u \rho+3 u_{x}+4 \mu^{3}=\theta .
$$

Using the same expansions as above

$$
\rho=\mu+\sum_{n=0}^{\infty} \rho_{n}(-2 \mu)^{-n}, \quad \theta=\sum_{n=0}^{\infty} \theta_{n}(-2 \mu)^{-n},
$$

one can obtain from (2.9) the following recursion relation:

$$
\begin{aligned}
\rho_{n+2}= & 2 u \rho_{n}+2 \sum_{i=0}^{n+1} \rho_{i} \rho_{n-i+1}-\frac{4}{3} \sum_{i, j=0}^{n} \rho_{i} \rho_{j} \rho_{n-i-j}-\frac{1}{3} \theta_{n} \\
& +2 D_{x}\left(\rho_{n+1}-\sum_{i=0}^{n} \rho_{i} \rho_{n-i}\right)-\frac{4}{3} D_{x}^{2} \rho_{n}-u \delta_{n,-1}+u_{1} \delta_{n 0}, \quad n=-2,-1,0, \ldots,
\end{aligned}
$$

where $\delta_{i, k}$ is the Kronecker delta. The obtained relation provides $\rho_{0}=0, \rho_{1}=-u, \rho_{2}=$ $-u_{1}-\theta_{0} / 3$, etc. As $D_{t} \rho_{0}=D_{x} \theta_{0}$ and $\rho_{0}=0$, then $\theta_{0}=0$. The higher canonical densities $\rho_{n}$, 
$n>2$ depend on $\theta_{n-2}$. The fluxes $\theta_{n}$ must be defined now from equations (1.4). For example, $\theta_{1}=u_{2}-3 u^{2}$.

The traditional method to obtain the canonical densities for an evolution system [25]

$$
\boldsymbol{u}_{t}=\boldsymbol{K}\left(\boldsymbol{u}, \boldsymbol{u}_{x}, \ldots, u_{n}\right), \quad \boldsymbol{u}(t, x) \in \mathbb{R}^{m}, \quad m \geqslant 1, \quad u_{k}^{\alpha}=\partial_{x}^{k} u^{\alpha} .
$$

consists, briefly, in the following. The main idea is to use the linearized equation

$$
\left(D_{t}-\boldsymbol{K}_{*}\right) \psi=0
$$

or its adjoint

$$
\left(D_{t}+\boldsymbol{K}_{*}^{+}\right) \varphi=0
$$

as the temporal Lax equation. Here

$$
\begin{array}{ll}
\left(\boldsymbol{K}_{*} \psi\right)^{\alpha}=\sum_{n, \beta} \frac{\partial K^{\alpha}}{\partial u_{n}^{\beta}} D_{x}^{n} \psi^{\beta}, & \left(\boldsymbol{K}_{*}^{+} \varphi\right)_{\alpha}=\sum_{n, \beta}\left(-D_{x}\right)^{n} \frac{\partial K^{\beta}}{\partial u_{n}^{\alpha}} \varphi_{\beta}, \\
D_{t}=\frac{\partial}{\partial t}+\sum_{n, \alpha} D_{x}^{n}\left(K^{\alpha}\right) \frac{\partial}{\partial u_{n}^{\alpha}}, & D_{x}=\frac{\partial}{\partial x}+\sum_{n, \alpha} u_{n+1}^{\alpha} \frac{\partial}{\partial u_{n}^{\alpha}} .
\end{array}
$$

The spatial Lax operator (formal symmetry) was introduced in [25] as the infinite operator series

$$
R=\sum_{k=-\infty}^{N} R_{k} D_{x}^{k}, \quad N>0
$$

commuting with $D_{t}-\boldsymbol{K}_{*}$. $R_{k}$ are matrix coefficients depending on $\boldsymbol{u}, \boldsymbol{u}_{x}, \ldots$ It was shown that $\operatorname{Tr}$ res $R$ (res $R=R_{-1}$ ) is the conserved density for system (2.10). Canonical densities have been defined by the formulas

$$
\rho_{n}=\operatorname{Tr} \operatorname{res} R^{n}, \quad n=1,2, \ldots,
$$

see [26] for details.

Operations with operator series (2.13) are not so simple, therefore we use an alternative method for obtaining the canonical densities. It was proposed in [32] heuristically and we present the following explanation (see also [33]).

Observation. One can obtain equation (2.9) from (2.2) by the following substitution

$$
\psi=e^{\omega}, \quad \omega=\int \rho d x+\theta d t
$$

where $\rho d x+\theta d t$ is the smooth closed 1-form, that is, $D_{t} \rho=D_{x} \theta$. This implies $e^{-\omega} D_{t} e^{\omega}=D_{t}+\theta$, $e^{-\omega} D_{x} e^{\omega}=D_{x}+\rho$ and so (2.9) follows. Another way to obtain the same equation is to prolong the operators $D_{t} \rightarrow \partial_{t}+\theta, D_{x} \rightarrow \partial_{x}+\rho$ in (2.2) formally and to set $\psi=1$. For systems, one must set $\psi_{\alpha}=1$ for a fixed $\alpha$ only.

We shall apply this method to system (1.1) now.

The linearized system (1.1) with prolonged operators $D_{x} \rightarrow D_{x}+\rho, D_{t} \rightarrow D_{t}+\theta$ takes the following form:

$$
\begin{aligned}
& {\left[\left(D_{x}+\rho\right)^{3}+F_{u}+F_{u_{1}}\left(D_{x}+\rho\right)+F_{u_{2}}\left(D_{x}+\rho\right)^{2}-D_{t}-\theta\right] \Psi_{1}} \\
& \quad+\left[F_{v}+F_{v_{1}}\left(D_{x}+\rho\right)+F_{v_{2}}\left(D_{x}+\rho\right)^{2}\right] \Psi_{2}=0
\end{aligned}
$$




$$
\begin{aligned}
& {\left[G_{u}+G_{u_{1}}\left(D_{x}+\rho\right)+G_{u_{2}}\left(D_{x}+\rho\right)^{2}\right] \Psi_{1}+a\left(D_{x}+\rho\right)^{3} \Psi_{2}} \\
& \quad+\left[G_{v}+G_{v_{1}}\left(D_{x}+\rho\right)+G_{v_{2}}\left(D_{x}+\rho\right)^{2}-D_{t}-\theta\right] \Psi_{2}=0 .
\end{aligned}
$$

If one sets here $\Psi_{1}=1$, then the first equation takes the following form

$$
\begin{aligned}
& \left(D_{x}+\rho\right)^{2} \rho+F_{u}+F_{u_{1}} \rho+F_{u_{2}}\left(D_{x}+\rho\right) \rho-\theta \\
& \quad+\left[F_{v}+F_{v_{1}}\left(D_{x}+\rho\right)+F_{v_{2}}\left(D_{x}+\rho\right)^{2}\right] \Psi_{2}=0 .
\end{aligned}
$$

It is obvious from this equation that the following forms of the asymptotic expansions are acceptable:

$$
\rho=\mu^{-1}+\sum_{n=0}^{\infty} \rho_{n} \mu^{n}, \quad \theta=\mu^{-3}+\sum_{n=0}^{\infty} \theta_{n} \mu^{n}, \quad \Psi_{2}=\sum_{n=0}^{\infty} \rho_{n} \mu^{n} .
$$

Here $\mu$ is a complex parameter. Then, after some simple calculations, the following recursion relations are obtained $(n \geqslant-1)$ :

$$
\begin{aligned}
& \rho_{n+2}=\frac{1}{3} \theta_{n}-\sum_{i=0}^{n+1} \rho_{i} \rho_{n-i+1}-\frac{1}{3} \sum_{i+j=0}^{n} \rho_{i} \rho_{j} \rho_{n-i-j}-\frac{1}{3} F_{u_{1}}\left(\delta_{n,-1}+\rho_{n}\right)-\frac{1}{3} F_{u} \delta_{n, 0} \\
& -\frac{1}{3}\left(F_{v}+F_{v_{1}} D_{x}+F_{v_{2}} D_{x}^{2}\right) \varphi_{n}-\frac{1}{3} F_{u_{2}}\left(D_{x} \rho_{n}+2 \rho_{n+1}+\sum_{i=0}^{n} \rho_{i} \rho_{n-i}\right) \\
& -\frac{1}{3} F_{v_{2}}\left(\varphi_{n+2}+2 \sum_{i=0}^{n} \rho_{i} \varphi_{n-i+1}+\sum_{i+j=0}^{n} \rho_{i} \rho_{j} \varphi_{n-i-j}\right) \\
& -\frac{1}{3} F_{v_{2}}\left(2 D_{x} \varphi_{n+1}+\sum_{i=0}^{n} \rho_{i} D_{x} \varphi_{n-i}+D_{x} \sum_{i=0}^{n} \rho_{i} \varphi_{n-i}\right) \\
& -D_{x}\left[\rho_{n+1}+\frac{1}{3} D_{x} \rho_{n}+\frac{1}{2} \sum_{i=0}^{n} \rho_{i} \rho_{n-i}\right]-\frac{1}{3} F_{v_{1}}\left(\varphi_{n+1}+\sum_{i=0}^{n} \rho_{i} \varphi_{n-i}\right), \\
& (1-a) \varphi_{n+3}=G_{u} \delta_{n, 0}+G_{u_{2}}\left(D_{x} \rho_{n}+2 \rho_{n+1}+\sum_{i=0}^{n} \rho_{i} \rho_{n-i}\right)+G_{u_{1}}\left(\delta_{n,-1}+\rho_{n}\right) \\
& -\sum_{i=0}^{n} \theta_{i} \varphi_{n-i}+G_{v} \varphi_{n}+G_{v_{1}}\left(D_{x} \varphi_{n}+\varphi_{n+1}+\sum_{i=0}^{n} \rho_{i} \varphi_{n-i}\right)-D_{t} \varphi_{n} \\
& +G_{v_{2}}\left(2 D_{x} \varphi_{n+1}+\sum_{i=0}^{n} \rho_{i} D_{x} \varphi_{n-i}+D_{x} \sum_{i=0}^{n} \rho_{i} \varphi_{n-i}\right) \\
& +G_{v_{2}}\left(\varphi_{n+2}+D_{x}^{2} \varphi_{n}+2 \sum_{i=0}^{n+1} \rho_{i} \varphi_{n-i+1}+\sum_{i+j=0}^{n} \rho_{i} \rho_{j} \varphi_{n-i-j}\right) \\
& +a D_{x}^{3} \varphi_{n}+3 a D_{x}^{2} \varphi_{n+1}+6 a \sum_{i=0}^{n+1} \rho_{i} D_{x} \varphi_{n-i+1}+3 a \sum_{i=0}^{n+2} \rho_{i} \varphi_{n-i+2} \\
& +3 a D_{x} \varphi_{n+2}+3 a \sum_{i+j=0}^{n} \rho_{i} \rho_{j} D_{x} \varphi_{n-i-j}+3 a \sum_{i=0}^{n} \varphi_{n-i+1} D_{x} \rho_{i} \\
& +\frac{3}{2} a \sum_{i+j=0}^{n} \varphi_{n-i-j} D_{x}\left(\rho_{i} \rho_{j}\right)+3 a \sum_{i+j=0}^{n+1} \rho_{i} \rho_{j} \varphi_{n-i-j+1}
\end{aligned}
$$




$$
+3 a D_{x} \sum_{i=0}^{n} \rho_{i} \varphi_{n-i}+a \sum_{i=0}^{n} \varphi_{n-i} D_{x}^{2} \rho_{i}+a \sum_{i+j+k=0}^{n} \rho_{i} \rho_{j} \rho_{k} \varphi_{n-i-j-k} .
$$

Here $\delta_{i, k}$ is the Kronecker delta, $F_{u_{1}}=\partial F / \partial u_{1}$ and so on. From the recursion relations it is obvious why the value $a=1$ is singular. Some of initial elements of the sequence $\left\{\rho_{n}, \varphi_{n}\right\}$ read

$$
\rho_{0}=-\frac{1}{3} F_{u_{2}}, \quad \varphi_{0}=0, \quad \varphi_{1}=\frac{1}{1-a} G_{u_{2}}
$$

others are introduced via the $\delta$-symbols.

If one sets in (2.15) $\Psi_{2}=1$ and $a \neq 0$, then one more pair of recursion relations for $\left\{\tilde{\rho}_{n}, \tilde{\varphi}_{n}\right\}$ is obtained. These recursion relations give us any desired number of canonical densities. As an example, we present here some more canonical densities:

$$
\begin{array}{ll}
\rho_{0}=-\frac{1}{3} F_{u_{2}}, & \rho_{1}=\frac{1}{9} F_{u_{2}}^{2}-\frac{1}{3} F_{u_{1}}+\frac{1}{3 b} F_{v_{2}} G_{u_{2}}+\frac{1}{3} D_{x} F_{u_{2}}, \\
\tilde{\rho}_{0}=-\frac{1}{3 a} G_{v_{2}}, & \tilde{\rho}_{1}=\frac{1}{9 a^{2}} G_{v_{2}}^{2}-\frac{1}{3 a} G_{v_{1}}-\frac{1}{3 a b} F_{v_{2}} G_{u_{2}}+\frac{1}{3 a} D_{x} G_{v_{2}},
\end{array}
$$

where $b=a-1$. The tilde denotes another sequence of canonical densities. Further canonical densities are too cumbersome, therefore we do not present them here.

To simplify investigation of the integrability conditions, an additional requirement is always imposed. This is the existence of a formal conservation law [25, 26]. A formal conservation law is an operator series $N$ in powers of $D_{x}^{-1}$. An equation for the formal conservation law can be written in the following operator form

$$
\left(D_{t}-K_{*}\right) \mathcal{N}=\mathcal{N}\left(D_{t}+K_{*}^{+}\right) .
$$

The form of this equation coincides with the form of the equation for the Noether operator [34]. That is a formal conservation law may be called a formal Noether operator.

If $\left(D_{t}-K_{*}, L\right)$ is the Lax pair for an equation, then $\left(D_{t}+K_{*}{ }^{+}, L^{+}\right)$is obviously the Lax pair for the same equation. Hence, canonical densities obtained from (2.11) must be equivalent to canonical densities obtained from (2.12).

It was shown in [21] that the first sequence of the canonical densities $\rho_{n}$ for system (1.1) obtained from (2.11) is equivalent to the first sequence of the canonical densities $\tau_{n}$ obtained from (2.12) and the second sequence of the canonical densities $\tilde{\rho}_{n}$ is equivalent to the second sequence of the canonical densities $\tilde{\tau}_{n}$. Hence, $\rho_{n}-\tau_{n} \in \operatorname{Im} D_{x}$ and $\tilde{\rho}_{n}-\tilde{\tau}_{n} \in \operatorname{Im} D_{x}$, or

$$
E_{\alpha}\left(\rho_{n}-\tau_{n}\right)=0, \quad E_{\alpha}\left(\tilde{\rho}_{n}-\tilde{\tau}_{n}\right)=0, \quad \alpha=1,2, \quad n=0,1,2, \ldots
$$

Equations (1.4) (or (1.5)) and (2.18) are said to be the necessary conditions of integrability. We shall refer to it simply as the integrability conditions for brevity.

Our computations have shown that

$$
\tau_{0}=-\rho_{0}, \quad \tilde{\tau}_{0}=-\tilde{\rho}_{0}, \quad \tau_{1}=\rho_{1}, \quad \tilde{\tau}_{1}=\tilde{\rho}_{1} .
$$

Other "adjoint" canonical densities $\tau_{i}$ and $\tilde{\tau}_{k}$ essentially differ from the "main" canonical densities $\rho_{i}$ and $\tilde{\rho}_{k}$. All canonical densities can be obtained using the Maple routines $c d$ and acd from the package JET (see [36]). These routines generate the "main" and the "adjoint" canonical densities, correspondingly, for almost any evolutionary system (an exclusion is the case of multiple roots of the main matrix of the system under consideration).

Thus, according to (2.16) and (2.19) we have $F_{u_{2}} \in \operatorname{Im} D_{x}$ and $G_{v_{2}} \in \operatorname{Im} D_{x}(a \neq 0)$. This implies the following lemma. 
Lemma 1. System (1.1) with $a(a-1) \neq 0$ satisfying the zeroth integrability conditions (2.18) reads

$$
\begin{aligned}
& u_{t}=u_{3}-\frac{3}{2 f} u_{2} D_{x} f+\frac{3}{4 f} f_{u_{1}} u_{2}^{2}+F_{1}\left(u, v, u_{1}, v_{1}, v_{2}\right), \\
& v_{t}=a v_{3}-\frac{3 a}{2 g} v_{2} D_{x} g+\frac{3 a}{4 g} g_{v_{1}} v_{2}^{2}+G_{1}\left(u, v, u_{1}, v_{1}, u_{2}\right) .
\end{aligned}
$$

where $\operatorname{ord}(f, g) \leqslant 1$.

Indeed, one may set $F_{u_{2}}=-3 / 2 D_{x} \ln f$ and $G_{v_{2}}=-3 / 2 a D_{x} \ln g$, where ord $(f, g) \leqslant 1$ because $\operatorname{ord}(F, G) \leqslant 2$. Then equations $(2.20)$ follow.

From higher integrability conditions one more lemma follows.

Lemma 2. Suppose system (2.20) is irreducible and satisfies the following eight integrability conditions $\rho_{2}-\tau_{2} \in \operatorname{Im} D_{x}, \tilde{\rho}_{2}-\tilde{\tau}_{2} \in \operatorname{Im} D_{x}$ and $D_{t} \rho_{n} \in \operatorname{Im} D_{x}, D_{t} \tilde{\rho}_{n} \in \operatorname{Im} D_{x}$, where $n=1,3,5$. Then the system must have the following form

$$
\begin{aligned}
& u_{t}=u_{3}-\frac{3}{2 f} u_{2} D_{x} f+\frac{3}{4 f} f_{u_{1}} u_{2}^{2}+f_{1} v_{2}^{2}+f_{2} v_{2}+f_{3}, \\
& v_{t}=a v_{3}-\frac{3 a}{2 g} v_{2} D_{x} g+\frac{3 a}{4 g} g_{v_{1}} v_{2}^{2}+g_{1} u_{2}^{2}+g_{2} u_{2}+g_{3}, \quad a \neq 0,
\end{aligned}
$$

where $\operatorname{ord}\left(f, g, f_{i}, g_{j}\right) \leqslant 1$.

A scheme of the proof has been presented in [21].

\section{List of integrable systems}

As it is shown in Section 2 the problem of the classification of integrable systems (1.1) is reduced to investigation of system (2.21). That is why it is necessary to start by investigating its symmetry properties.

Lemma 3. System (2.21) are invariant under any point transformation of the form
(a) $t^{\prime}=\alpha^{3} t+\beta$
$x^{\prime}=\alpha x+\gamma t+\delta$,
$\alpha \neq 0$,
$u^{\prime}=u$
$v^{\prime}=v$,
(b) $u^{\prime}=h_{1}(u), \quad v^{\prime}=h_{2}(v)$,

and under the following permutation transformation

(c) $t^{\prime}=a t, \quad u^{\prime}=v, \quad v^{\prime}=u$,

where $\alpha, \beta, \gamma$ and $\delta$ are constants, $h_{i}$ are arbitrary smooth functions.

The classification of systems of type (2.21) has been performed by modulo of the presented transformations.

Moreover, some systems (2.21) admit invertible contact transformations. An effective tool for searching such contact transformations is investigation of the canonical conserved densities. For example, system (3.24) from the next section has the first canonical conserved density of the following form:

$$
\rho_{1}=\left(v_{1}-\frac{2}{3} u e^{v}\right)^{2}+2 c_{1}^{2} e^{-2 v} .
$$

It is obvious that the best variables for that system are

$$
U=e^{-v} \quad \text { and } \quad V=v_{1}-\frac{2}{3} u e^{v} .
$$


This is an invertible contact transformation. In terms of $U$ and $V$ the system takes the following simple form:

$$
\begin{aligned}
& U_{t}=D_{x}\left(U_{2}+\frac{3}{2} U V_{1}-\frac{3}{4} U V^{2}+\frac{1}{2} c_{1}^{2} U^{3}\right), \\
& V_{t}=\frac{1}{4} D_{x}\left(V^{3}-2 V_{2}\right)-\frac{3}{2} c_{1}^{2} D_{x}\left(2 U U_{1}+U^{2} V\right) .
\end{aligned}
$$

If $c_{1} \neq 0$ this system can be reduced to (3.10) by scaling, otherwise the system is triangular: the equation for $V$ will be independent single $\mathrm{mKdV}$. Moreover, the equation for $U$ becomes linear. That is why $c_{1} \neq 0$ in (3.24).

Canonical densities for the triangular systems contain only one highest order term in the second power as in the considered example $\rho=V^{2}$ or $\rho=V_{x}^{2}+\cdots$, or $\rho=V_{x x}^{2}+\cdots$ etc. Triangular systems and those reducible to the triangular form have been omitted in the classification process as trivial.

To classify integrable systems (1.1) with $a(a-1) \neq 0$ one must solve a huge number of large overdetermined partial differential systems for eight unknown functions of four variables. This work has required powerful computers and has taken about six years. All the calculations have been performed in the interactive mode of operation because automatic solving of large systems of partial differential equations is still impossible. The package PDSOLVE from the excellent system Maple makes errors solving some single partial differential equations. The package DIFFALG cannot operate with large systems because its algorithms are too cumbersome. Thus, one has to solve complicated problems in the interactive mode. Hence, to obtain a true solution one must enter true data! Under such circumstances errors are probable. The longer the computations the more probable are errors. This is the reason why we cannot state with confidence that all computations have been precise all these six years. That is why the statement on completeness of the obtained set of integrable systems is formulated as a hypothesis.

In this and in the following sections $c, c_{i}, k, k_{i}$ are arbitrary constants.

Hypothesis. Suppose system (2.21) with $a=-1 / 2$ is irreducible. If the system has infinitely many canonical conservation laws, then it can be reduced by an appropriate point transformation to one of the following systems:

$$
\begin{aligned}
& u_{t}=u_{3}+v u_{1}, \quad v_{t}=-\frac{1}{2} v_{3}+u u_{1}-v v_{1} \\
& u_{t}=u_{3}+v_{1} u_{1}, \quad v_{t}=-\frac{1}{2} v_{3}+\frac{1}{2}\left(u^{2}-v_{1}^{2}\right) \\
& u_{t}=u_{3}+v u_{1}, \quad v_{t}=-\frac{1}{2} v_{3}-v v_{1}+u_{1} \\
& u_{t}=u_{3}+v u_{1}+v_{1} u, \quad v_{t}=-\frac{1}{2} v_{3}-v v_{1}+u \\
& u_{t}=u_{3}+v_{1} u_{1}, \quad v_{t}=-\frac{1}{2} v_{3}-\frac{1}{2} v_{1}^{2}+u \\
& u_{t}=u_{3}+u u_{1}+v_{1}, \quad v_{t}=-\frac{1}{2} v_{3}+\frac{3}{2} u_{1} u_{2}-u v_{1} \\
& u_{t}=u_{3}+v_{2}+k u_{1}, \quad v_{t}=-\frac{1}{2} v_{3}+\frac{3}{2} u u_{2}+\frac{3}{4} u_{1}^{2}+\frac{1}{3} u^{3}+k\left(u^{2}-v_{1}\right) \\
& u_{t}=u_{3}+\frac{3}{2} v v_{2}+\frac{3}{4} v_{1}^{2}+\frac{1}{3} v^{3}-k\left(v^{2}+u_{1}\right), \quad v_{t}=-\frac{1}{2} v_{3}+u_{2}+k v_{1} \\
& u_{t}=u_{3}-\frac{3}{2} u_{1} v_{2}-\frac{3}{4} u_{1} v_{1}^{2}+\frac{1}{4} u_{1}^{3}, \quad v_{t}=-\frac{1}{2} v_{3}+\frac{3}{2} u_{1} u_{2}-\frac{3}{4} u_{1}^{2} v_{1}+\frac{1}{4} v_{1}^{3} \\
& u_{t}=\left(u_{2}-\frac{3}{2} u u_{1}-\frac{3}{4} u v^{2}+\frac{1}{4} u^{3}\right)_{x}, \quad \frac{3}{2} v_{x} ; \\
& \left.u_{t}=u_{3}-\frac{3}{2} v_{2}-\frac{3}{2} u_{1} v_{1}-\frac{1}{2} u_{1}^{3}, \quad v_{2}+\frac{1}{2} u_{1}^{2}\right)^{2}-\frac{3}{4} v_{1}^{2} \\
& \left.\left.u_{t}=\left(u_{2}-\frac{3}{2} v_{1}-\frac{3}{2} u v-\frac{1}{2} u^{3}\right)_{x}, \quad v_{1} u^{2}\right)^{2}-\frac{3}{4} v^{2}\right)_{x} \\
& u_{t}=u_{3}-3 g v_{2}-3 u_{1}\left(u_{1}+v_{1}\right)-\frac{3}{2} v_{1}^{2}-6 v_{1} g^{2}-c_{1} g^{3}-3 g^{4}, \\
& v_{t}=-\frac{1}{2} v_{3}-\frac{3}{4} c_{1} u_{2}+3 u_{1}^{2}-\frac{3}{2} v_{1}^{2}-6 u_{1} g^{2}+c_{1} g^{3}+3 g^{4}, \quad g=u+v ; \\
& u_{t}=u_{3}-3 u_{1} v_{1}+\left(u-3 v^{2}\right) u_{1}, \quad v_{t}=-\frac{1}{2} v_{3}+\frac{1}{2} u_{2}-u_{1} v-\left(u-3 v^{2}\right) v_{1} \\
& u_{t}=u_{3}-3 u_{1} v_{2}+u u_{1}-3 u_{1} v_{1}^{2}, \quad v_{t}=-\frac{1}{2} v_{3}+\frac{1}{2} u_{1}-u v_{1}+v_{1}^{3} ;
\end{aligned}
$$




$$
\begin{aligned}
& u_{t}=u_{3}+\left(k+\sqrt{u^{2}+v_{1}}\right) u_{1} \\
& v_{t}=-\frac{1}{2} v_{3}+\frac{3}{8} \frac{\left(2 u u_{1}+v_{2}\right)^{2}}{u^{2}+v_{1}}-3 u u_{2}-k\left(2 u^{2}+v_{1}\right)-\frac{2}{3}\left(u^{2}+v_{1}\right)^{3 / 2} \text {; } \\
& u_{t}=u_{3}-\frac{3}{4} \frac{\left(2 v v_{1}+u_{2}\right)^{2}}{v^{2}+u_{1}}+3 v v_{2}+\frac{3}{2} v_{1}^{2}+\frac{2}{3} v^{3}-k\left(2 v^{2}+u_{1}\right), \\
& v_{t}=-\frac{1}{2} v_{3}+\frac{1}{2} u_{2}+k v_{1} \\
& u_{t}=u_{3}+\frac{u_{1}\left(u_{1}+v_{2}\right)}{\sqrt{u+v_{1}}}-\frac{4}{3} u_{1} v_{1}+c_{1} u_{1} \sqrt{u+v_{1}} \text {, } \\
& v_{t}=-\frac{1}{2} v_{3}-\frac{3}{2} u_{2}+\frac{3}{8} \frac{\left(u_{1}+v_{2}\right)^{2}}{u+v_{1}}+\frac{2}{3} v_{1}^{2}-\frac{4}{3} u^{2}-2 u_{1} \sqrt{u+v_{1}}-\frac{2}{3} c_{1}\left(u+v_{1}\right)^{3 / 2} ; \\
& u_{t}=u_{3}+u_{1} \sqrt{u+v_{1}}-k u_{1} \text {, } \\
& v_{t}=-\frac{1}{2} v_{3}-\frac{3}{2} u_{2}+\frac{3}{8} \frac{\left(u_{1}+v_{2}\right)^{2}}{u+v_{1}}-\frac{2}{3}\left(u+v_{1}\right)^{3 / 2}+2 k u+k v_{1} ; \\
& u_{t}=u_{3}+u v_{1}+\left(u^{2}+v\right) u_{1}, \quad v_{t}=-\frac{1}{2} v_{3}+3 u_{1} u_{2}-\left(u^{2}+v\right) v_{1} ; \\
& u_{t}=u_{3}+3(u+k) v_{2}+3 u_{1}\left(v_{1}+u^{2}\right) \text {, } \\
& v_{t}=-\frac{1}{2} v_{3}-\frac{3}{2} u u_{2}-\frac{3}{2}\left(v_{1}+u^{2}\right)^{2}-\frac{3}{4} u_{1}^{2}+k u^{3}+\frac{3}{4} u^{4} \text {; } \\
& u_{t}=u_{3}-\frac{3}{2} v_{2}-\frac{3}{2} u_{1} v_{1}-\frac{1}{2} u_{1}^{3}-3 u_{1}\left(c_{1} e^{u}+2 c_{2} e^{2 u}\right) \text {, } \\
& v_{t} v=-\frac{1}{2} v_{3}+\frac{3}{2}\left(\frac{1}{2} u_{1}^{2}-u_{2}+v_{1}+c_{1} e^{u}+2 c_{2} e^{2 u}\right)^{2} \\
& -\frac{3}{4} v_{1}^{2}-\frac{3}{2} c_{1} u_{2} e^{u}+\frac{3}{4} c_{1}^{2} e^{2 u}+2 c_{1} c_{2} e^{3 u}, \quad c_{1} \neq 0 \quad \text { or } \quad c_{2} \neq 0 ; \\
& u_{t}=u_{3}-\frac{3}{2} u_{1} v_{2}-\frac{3}{4} u_{1} v_{1}^{2}+u u_{1}-c^{2} u_{1} e^{-2 v}, \\
& v_{t}=-\frac{1}{2} v_{3}+\frac{1}{4} v_{1}^{3}+u_{1}-u v_{1}+c^{2} v_{1} e^{-2 v} \\
& u_{t}=u_{3}-\frac{3}{2} u_{1} v_{2}-\frac{3}{4} u_{1} v_{1}^{2}+u_{1} e^{v}\left(u_{1}+2 u v_{1}\right)-\frac{1}{3} u^{2} u_{1} e^{2 v}-\frac{3}{2} c_{1}^{2} u_{1} e^{-2 v} \text {, } \\
& v_{t}=-\frac{1}{2} v_{3}+\frac{v_{1}^{3}}{4}+u_{2} e^{v}+\frac{1}{3} u e^{2 v}\left(2 u_{1}+u v_{1}\right)+\frac{3}{2} c_{1}^{2} v_{1} e^{-2 v}, \quad c_{1} \neq 0 ; \\
& u_{t}=u_{3}+3 u_{2} v_{1}+\frac{3}{2} u_{1} v_{2}+\frac{9}{4} u_{1} v_{1}^{2}-u u_{1} e^{2 v}-e^{-3 v} \text {, } \\
& v_{t}=-\frac{1}{2} v_{3}+\frac{1}{4} v_{1}^{3}+\left(u_{1}+u v_{1}\right) e^{2 v} \\
& u_{t}=u_{3}+3 u_{2} v_{1}+\frac{3}{2} u_{1} v_{2}+\frac{9}{4} u_{1} v_{1}^{2}-u u_{1} e^{2 v}-\frac{1}{4} u_{1} e^{-2 v} \text {, } \\
& v_{t}=-\frac{1}{2} v_{3}+\frac{1}{4} v_{1}^{3}+\left(u_{1}+u v_{1}\right) e^{2 v}+\frac{1}{4} v_{1} e^{-2 v} \text {; } \\
& u_{t}=u_{3}+\frac{3}{2} u_{1} v_{2}+3 u_{2} v_{1}+\frac{9}{4} u_{1} v_{1}^{2}-c_{1}^{2} u_{1} e^{-2 v}-\frac{1}{2} u_{1} e^{2 v}\left(u^{2}+c_{2}\right) \text {, } \\
& v_{t}=-\frac{1}{2} v_{3}+\frac{1}{4} v_{1}^{3}+c_{1}^{2} v_{1} e^{-2 v}+\frac{1}{2} e^{2 v}\left(2 u u_{1}+u^{2} v_{1}+c_{2} v_{1}\right) ; \\
& u_{t}=u_{3}+\frac{3}{2} u_{1} v_{2}+3 u_{2} v_{1}+\frac{9}{4} u_{1} v_{1}^{2}-\frac{1}{3} e^{2 v}\left(u_{1}\left(6 u^{2}+c_{1}\right)+4 u v_{1}\left(2 u^{2}+c_{1}\right)\right) \\
& +e^{v}\left(v_{2}\left(2 u^{2}+c_{1}\right)+\left(u_{1}+2 u v_{1}\right)^{2}+2 c_{1} v_{1}^{2}\right) \text {, } \\
& v_{t}=-\frac{1}{2} v_{3}+\frac{1}{4} v_{1}^{3}+\frac{1}{3} e^{2 v}\left(4 u u_{1}+\left(6 u^{2}+c_{1}\right) v_{1}\right)+e^{v}\left(u_{2}+2 u_{1} v_{1}\right) ; \\
& u_{t}=u_{3}+\frac{3}{2} u_{1} v_{2}+3 u_{2} v_{1}+\frac{9}{4} u_{1} v_{1}^{2}+3 u v_{2}\left(c_{1} u e^{v}+c_{2}\right)+c_{1}\left(c_{1}^{2}-1\right) u^{4} e^{3 v} \\
& -\frac{3}{4} u^{2} e^{2 v}\left(u_{1}\left(1+5 c_{1}^{2}\right)+8 c_{1}^{2} u v_{1}+2 c_{2} u\left(1-3 c_{1}^{2}\right)\right)-3 c_{2}^{2}\left(u_{1}+2 u v_{1}\right) \\
& +\frac{3}{2} c_{1} e^{v}\left(u_{1}+2 u v_{1}-2 c_{2} u\right)^{2}+\frac{3}{2} c_{2} v_{1}\left(2 u_{1}+3 u v_{1}\right) \text {, } \\
& v_{t}=-\frac{1}{2} v_{3}+\frac{1}{4} v_{1}^{3}+\frac{3}{2} c_{1} e^{v}\left(u_{2}+2 u_{1} v_{1}\right)+c_{1}\left(1-c_{1}^{2}\right) u^{3} e^{3 v}+6 c_{1} c_{2} u e^{v}\left(v_{1}-c_{2}\right) \\
& +\frac{3}{4} u e^{2 v}\left(2 u_{1}\left(1+c_{1}^{2}\right)+u v_{1}\left(1+5 c_{1}^{2}\right)+2 c_{2} u\left(1-3 c_{1}^{2}\right)\right)+\frac{3}{2} c_{2} v_{1}\left(2 c_{2}-v_{1}\right) \text {; } \\
& u_{t}=u_{3}+\frac{3}{2} u_{1} v_{2}+3 u_{2} v_{1}+\frac{9}{4} u_{1} v_{1}^{2}+3 e^{v}\left(u^{2}+c\right)\left(v_{2}+2 v_{1}^{2}\right)+\frac{3}{2} e^{v} u_{1}\left(u_{1}+4 u v_{1}\right) \\
& -\frac{3}{2} e^{2 v}\left(\left(3 u^{2}+c\right) u_{1}+4\left(u^{2}+c\right) u v_{1}\right) \text {, } \\
& v_{t}=-\frac{1}{2} v_{3}+\frac{1}{4} v_{1}^{3}+\frac{3}{2} e^{v}\left(u_{2}+2 u_{1} v_{1}\right)+\frac{3}{2} e^{2 v}\left(2 u u_{1}+\left(3 u^{2}+c\right) v_{1}\right) \text {; }
\end{aligned}
$$




$$
\begin{aligned}
& u_{t}=u_{3}-\frac{3}{2} u_{1} v_{2}-\frac{3}{4} u_{1} v_{1}^{2}+\frac{1}{4} u_{1}^{3}-c_{1} e^{-2 v} u_{1}-c_{2}\left(u_{1}+2 v_{1}\right) e^{2(u+v)} \\
& +c_{3}\left(u_{1}-2 v_{1}\right) e^{2(v-u)} \text {, } \\
& v_{t}=-\frac{1}{2} v_{3}+\frac{3}{2} u_{1} u_{2}-\frac{3}{4} u_{1}^{2} v_{1}+\frac{1}{4} v_{1}^{3}+c_{1} e^{-2 v} v_{1}+\left(c_{2} e^{2(u+v)}-c_{3} e^{2(v-u)}\right) v_{1} ; \\
& u_{t}=u_{3}-\frac{3}{2} u_{1} v_{2}-\frac{3}{4} u_{1} v_{1}^{2}+\frac{1}{4} u_{1}^{3}+\left(c_{2} e^{u}+c_{3} e^{-u}-3 c_{1}^{2} e^{-2 v}\right) u_{1} \text {, } \\
& v_{t}=-\frac{1}{2} v_{3}+\frac{3}{2} u_{1} u_{2}-\frac{3}{4} u_{1}^{2} v_{1}+\frac{1}{4} v_{1}^{3}+\left(c_{2} e^{u}-c_{3} e^{-u}\right) u_{1} \\
& +\left(3 c_{1}^{2} e^{-2 v}-c_{2} e^{u}-c_{3} e^{-u}\right) v_{1} \text {; } \\
& u_{t}=u_{3}-\frac{3}{2} u_{1} v_{2}-\frac{3}{4} u_{1} v_{1}^{2}+\frac{1}{4} u_{1}^{3}+3 k\left(u_{1}^{2}-2 v_{2}\right) e^{-v}-3\left(c^{2}-3 k^{2}\right) u_{1} e^{-2 v} \\
& +8 k\left(k^{2}-c^{2}\right) e^{-3 v}, \\
& v_{t}=-\frac{1}{2} v_{3}+\frac{3}{2} u_{1} u_{2}-\frac{3}{4} u_{1}^{2} v_{1}+\frac{1}{4} v_{1}^{3}+3 k\left(u_{2}-2 u_{1} v_{1}\right) e^{-v}+3\left(c^{2}-3 k^{2}\right) v_{1} e^{-2 v} \text {; } \\
& u_{t}=u_{3}-\frac{3}{2} u_{1} v_{2}-\frac{3}{4} u_{1} v_{1}^{2}+\frac{1}{4} u_{1}^{3}-3 c_{1}^{2} u_{1} e^{2(u+v)}+3 c_{1} u_{1}\left(u_{1}+2 v_{1}\right) e^{u+v} \\
& +\left(c_{2} e^{-u}-3 c_{3}^{2} e^{-2 v}\right) u_{1} \text {, } \\
& v_{t}=-\frac{1}{2} v_{3}+\frac{3}{2} u_{1} u_{2}-\frac{3}{4} u_{1}^{2} v_{1}+\frac{1}{4} v_{1}^{3}+3 c_{1}^{2}\left(2 u_{1}+v_{1}\right) e^{2(u+v)}+3 c_{1}\left(u_{2}+u_{1}^{2}\right) e^{u+v} \\
& -c_{2}\left(u_{1}+v_{1}\right) e^{-u}+3 c_{3}^{2} v_{1} e^{-2 v} \text {; } \\
& u_{t}=u_{3}-\frac{3}{2} u_{1} v_{2}-\frac{3}{4} u_{1} v_{1}^{2}+\frac{1}{4} u_{1}^{3}+3 c_{2} u_{1}\left(u_{1}+2 v_{1}\right) e^{u+v}-4 c_{1} c_{2} e^{3(u+v)} \\
& +3\left[\left(c_{1}-c_{2}^{2}\right) u_{1}+2 c_{1} v_{1}\right] e^{2(u+v)}, \\
& v_{t}=-\frac{1}{2} v_{3}+\frac{3}{2} u_{1} u_{2}-\frac{3}{4} u_{1}^{2} v_{1}+\frac{1}{4} v_{1}^{3}+3 c_{2}\left(u_{2}+u_{1}^{2}\right) e^{u+v}+4 c_{1} c_{2} e^{3(u+v)} \\
& +3\left[2 c_{2}^{2} u_{1}-\left(c_{1}-c_{2}^{2}\right) v_{1}\right] e^{2(u+v)} \\
& u_{t}=u_{3}-\frac{3}{2} u_{1} v_{2}-\frac{3}{4} u_{1} v_{1}^{2}+\frac{1}{4} u_{1}^{3}+\frac{2}{3} c_{1}^{2} u_{1} e^{-2 v}+c_{1}\left(2 v_{2}-u_{1}^{2}\right) e^{-v} \\
& -2 c_{1} c_{2}\left(u_{1}+2 v_{1}\right) e^{u}+3 c_{2} u_{1}\left(u_{1}+2 v_{1}\right) e^{u+v}-3 c_{2}^{2} u_{1} e^{2(u+v)}, \\
& v_{t}=-\frac{1}{2} v_{3}+\frac{3}{2} u_{1} u_{2}-\frac{3}{4} u_{1}^{2} v_{1}+\frac{1}{4} v_{1}^{3}-\frac{2}{3} c_{1}^{2} v_{1} e^{-2 v}+c_{1}\left(2 u_{1} v_{1}-u_{2}\right) e^{-v} \\
& -2 c_{1} c_{2}\left(u_{1}-v_{1}\right) e^{u}+3 c_{2}\left(u_{2}+u_{1}^{2}\right) e^{u+v}+3 c_{2}^{2}\left(2 u_{1}+v_{1}\right) e^{2(u+v)} \text {; } \\
& u_{t}=u_{3}-\frac{3}{2} u_{1} v_{2}-\frac{3}{4} u_{1} v_{1}^{2}+\frac{1}{4} u_{1}^{3}-3 u_{1}\left[c_{1}^{2} e^{2(u+v)}+c_{2}^{2} e^{2(v-u)}+2 c_{1} c_{2} e^{2 v}\right] \\
& -3 c_{3}^{2} u_{1} e^{-2 v}+3 c_{1} u_{1}\left(u_{1}+2 v_{1}\right) e^{u+v}-3 c_{2} u_{1}\left(u_{1}-2 v_{1}\right) e^{v-u}, \\
& v_{t}=-\frac{1}{2} v_{3}+\frac{3}{2} u_{1} u_{2}-\frac{3}{4} u_{1}^{2} v_{1}+\frac{1}{4} v_{1}^{3}+3 c_{1}^{2}\left(2 u_{1}+v_{1}\right) e^{2(u+v)}+6 c_{1} c_{2} v_{1} e^{2 v} \\
& +3 c_{2}^{2}\left(v_{1}-2 u_{1}\right) e^{2(v-u)}+3 c_{1}\left(u_{2}+u_{1}^{2}\right) e^{u+v}+3 c_{2}\left(u_{1}^{2}-u_{2}\right) e^{v-u}+3 c_{3}^{2} v_{1} e^{-2 v} \text {; } \\
& u_{t}=u_{3}-\frac{3}{2} u_{1} v_{2}-\frac{3}{4} u_{1} v_{1}^{2}+\frac{1}{4} u_{1}^{3}-6 c_{1}^{3} e^{3(u+v)}-\frac{3}{4} c_{1}^{2}\left(5 u_{1}-8 v_{1}\right) e^{2(u+v)} \\
& +\frac{9}{2} c_{1} u_{1}\left(u_{1}+2 v_{1}\right) e^{u+v}+2 c_{1}^{2} c_{2} e^{2 u+v}+\frac{2}{3} c_{1} c_{2}^{2} e^{u-v}-\frac{1}{2} c_{1} c_{2}\left(7 u_{1}+12 v_{1}\right) e^{u} \\
& +c_{2}\left(2 v_{2}-u_{1}^{2}\right) e^{-v}+\frac{11}{12} c_{2}^{2} u_{1} e^{-2 v}-\frac{2}{9} c_{2}^{3} e^{-3 v}, \\
& v_{t}=-\frac{1}{2} v_{3}+\frac{3}{2} u_{1} u_{2}-\frac{3}{4} u_{1}^{2} v_{1}+\frac{1}{4} v_{1}^{3}+6 c_{1}^{3} e^{3(u+v)}+\frac{3}{4} c_{1}^{2}\left(18 u_{1}+5 v_{1}\right) e^{2(u+v)} \\
& +\frac{9}{2} c_{1}\left(u_{2}+u_{1}^{2}\right) e^{u+v}-4 c_{1}^{2} c_{2} e^{2 u+v}+\frac{2}{3} c_{1} c_{2}^{2} e^{u-v}-\frac{7}{2} c_{1} c_{2}\left(u_{1}-v_{1}\right) e^{u} \\
& +c_{2}\left(2 u_{1} v_{1}-u_{2}\right) e^{-v}-\frac{11}{12} c_{2}^{2} v_{1} e^{-2 v} \text {; } \\
& u_{t}=u_{3}-\frac{3}{2} u_{1} v_{2}-\frac{3}{4} u_{1} v_{1}^{2}+\frac{1}{4} u_{1}^{3}+c_{3} e^{-v}\left(u_{1}^{2}-2 v_{2}\right)+\frac{2}{3} c_{3}^{2} u_{1} e^{-2 v} \\
& +c_{1}\left(3 u_{1} e^{u+v}+2 c_{3} e^{u}\right)\left(u_{1}+2 v_{1}\right)-c_{2}\left(3 u_{1} e^{v-u}+2 c_{3} e^{-u}\right)\left(u_{1}-2 v_{1}\right) \\
& -3 u_{1}\left(c_{1} e^{u}+c_{2} e^{-u}\right)^{2} e^{2 v}, \\
& v_{t}=-\frac{1}{2} v_{3}+\frac{3}{2} u_{1} u_{2}-\frac{3}{4} u_{1}^{2} v_{1}+\frac{1}{4} v_{1}^{3}+c_{3} e^{-v}\left(u_{2}-2 u_{1} v_{1}\right)-\frac{2}{3} c_{3}^{2} v_{1} e^{-2 v} \\
& +3 c_{1}^{2} e^{2(u+v)}\left(2 u_{1}+v_{1}\right)-3 c_{2}^{2} e^{2(v-u)}\left(2 u_{1}-v_{1}\right)+3 c_{1} e^{u+v}\left(u_{2}+u_{1}^{2}\right) \\
& -3 c_{2} e^{v-u}\left(u_{2}-u_{1}^{2}\right)+2 c_{2} c_{3} e^{-u}\left(u_{1}+v_{1}\right)+2 c_{1} c_{3} e^{u}\left(u_{1}-v_{1}\right)+6 c_{1} c_{2} v_{1} e^{2 v} \text {; }
\end{aligned}
$$




$$
\begin{aligned}
u_{t}= & u_{3}-\frac{3}{4} \frac{\left(2 g^{3}-u_{2}+2 g v_{1}\right)^{2}}{u_{1}-g^{2}}+3 g\left(u_{2}-v_{2}\right)-6 u_{1}^{2}-9 u_{1} v_{1}-\frac{3}{2} v_{1}^{2} \\
& -3\left(5 g^{2}+4 c g+c^{2}\right) u_{1}-6 g^{2} v_{1}+2 c g^{2}(8 g+3 c)+9 g^{4}, \\
v_{t}= & -\frac{1}{2} v_{3}+\frac{3}{4} \frac{\left(2 g^{3}-u_{2}+2 g v_{1}\right)^{2}}{u_{1}-g^{2}}-3(3 g+c) u_{2}-\frac{3}{2} v_{1}^{2}+3\left(9 g^{2}+8 c g+2 c^{2}\right) u_{1} \\
& +3\left(6 g^{2}+4 c g+c^{2}\right) v_{1}-2 c g^{2}(8 g+3 c)-9 g^{4}, \quad g=u+v ;
\end{aligned}
$$

Remark 1. Systems (3.1), (3.3), (3.6) and (3.20) were proposed in [3], where system (3.20) is given with a misprint. System (3.10) was presented in [29].

Remark 2. Ten pairs of integrability conditions (for $\rho_{0}-\rho_{9}$ and $\tilde{\rho}_{0}-\tilde{\rho}_{9}$ ) have been verified for each system (3.1)-(3.40), and nontrivial higher conserved densities with orders 2, 3, 4 and 5 have been found.

Remark 3. It is shown in [21] that systems (3.10) and (3.12) are unique divergent systems of the form (2.21) that satisfy the integrability conditions.

Remark 4. System (3.22) is a modification of (3.11), systems (3.31)-(3.39) are modifications of (3.9).

Remark 5. Canonical densities for system (3.25) depend on the nonlocal variable $w=D_{x}^{-1} e^{-v}$.

Remark 6. Many of the systems possess discrete symmetries. They are:

$$
\begin{aligned}
& u \rightarrow-u \text { for }(3.1),(3.2),(3.9),(3.10),(3.16),(3.20) \text { and }(3.27) ; \\
& u \rightarrow-u, v \rightarrow v+\pi i \text { for }(3.24) ; \\
& u \rightarrow i u, v \rightarrow v-\frac{i}{2} \pi, c_{1} \rightarrow-c_{1} \text { for }(3.28) ; \\
& \{u \rightarrow-u, v \rightarrow v+\pi i\} \cup\left\{v \rightarrow v+\pi i, c_{1} \rightarrow-c_{1}\right\} \cup\left\{u \rightarrow-u, c_{1} \rightarrow-c_{1}\right\} \text { for }(3.29) ; \\
& \{u \rightarrow-u, v \rightarrow v+\pi i\} \cup\left\{u \rightarrow i u, v \rightarrow v-\frac{i}{2} \pi\right\} \text { for }(3.30) ; \\
& u \rightarrow-u, c_{2} \rightarrow c_{3}, c_{3} \rightarrow c_{1} \text { for }(3.32) ; \\
& u \rightarrow-u, k \rightarrow-k \text { for }(3.33) ; \\
& u \rightarrow-u, c_{1} \rightarrow c_{2}, c_{2} \rightarrow c_{1} \text { for }(3.37) ; \\
& u \rightarrow-u, c_{3} \rightarrow-c_{3}, c_{2} \rightarrow c_{1}, c_{1} \rightarrow c_{2} \text { for }(3.39) .
\end{aligned}
$$

Also, systems (3.1), (3.2), (3.9), (3.10) and (3.27) preserve the real shape under the transformation $u \rightarrow i u$. System (3.33) keeps the real shape under the transformation $u \rightarrow i u, k \rightarrow i k$.

\subsection{Example of computations}

Let us consider the simplest case of system (1.1):

$$
u_{t}=u_{3}+f_{1}(u, v) u_{1}+f_{2}(u, v) v_{1}, \quad v_{t}=a v_{3}+g_{1}(u, v) u_{1}+g_{2}(u, v) v_{1},
$$

where $a(a-1) \neq 0$. Formulas $(2.16)$ are reduced now to the following

$$
\rho_{0}=0, \quad \tilde{\rho}_{0}=0, \quad \rho_{1}=-\frac{1}{3} f_{1}, \quad \tilde{\rho}_{1}=-\frac{1}{3 a} g_{2} .
$$

The further canonical densities read

$$
\begin{aligned}
& \rho_{2}=-\frac{1}{3}\left(f_{1, u} u_{1}+f_{2, u} v_{1}\right)+\frac{1}{3} D_{x} f_{1}, \quad \tilde{\rho}_{2}=-\frac{1}{3 a}\left(g_{1, v} u_{1}+g_{2, v} v_{1}\right)+\frac{1}{3 a} D_{x} g_{2}, \\
& \tau_{2}=\frac{1}{3}\left(f_{1, u} u_{1}+f_{2, u} v_{1}\right), \quad \tilde{\tau}_{2}=\frac{1}{3 a}\left(g_{1, v} u_{1}+g_{2, v} v_{1}\right),
\end{aligned}
$$

where indices after commas denote derivatives. 
The first integrability condition (1.5) for $\rho_{1}$ can be split with respect to $u_{3}, v_{3}, u_{2}$ and $v_{2}$. This provides the following equations

$$
f_{1, u v}=0, \quad f_{1, \text { uuu }}=0, \quad f_{1, v v v}=0, \quad \text { or } \quad f_{1}(u, v)=c_{1} u^{2}+c_{2} u+c_{3} v^{2}+c_{4} v+c_{5} .
$$

Analogously, condition (1.5) for $\tilde{\rho}_{1}$ implies $g_{2}(u, v)=b_{1} u^{2}+b_{2} u+b_{3} v^{2}+b_{4} v+b_{5}$. It is obvious from (3.43) that the second integrability conditions (2.18) are $\tau_{2} \in \operatorname{Im} D_{x}$ and $\tilde{\tau}_{2} \in \operatorname{Im} D_{x}$. These conditions provide $f_{2, u u}=g_{1, v v}=0$ or $f_{2}(u, v)=u f_{3}(v)+f_{4}(v), g_{1}(u, v)=v g_{3}(u)+g_{4}(u)$.

Thus, system (3.41) takes the following form:

$$
\begin{aligned}
& u_{t}=u_{3}+\left(c_{1} u^{2}+c_{2} u+c_{3} v^{2}+c_{4} v+c_{5}\right) u_{1}+\left(u f_{3}(v)+f_{4}(v)\right) v_{1}, \\
& v_{t}=a v_{3}+\left(v g_{3}(u)+g_{4}(u)\right) u_{1}+\left(b_{1} u^{2}+b_{2} u+b_{3} v^{2}+b_{4} v+b_{5}\right) v_{1} .
\end{aligned}
$$

Now one can obtain $\theta_{2}=D_{x}^{-1} D_{t} \rho_{2}$ and $\tilde{\theta}_{2}=D_{x}^{-1} D_{t} \tilde{\rho}_{2}$ in an explicit form. The expressions $D_{t} \rho_{1}$ and $D_{t} \tilde{\rho}_{1}$ are not the total derivatives yet:

$$
\begin{aligned}
& D_{t} \rho_{1}=D_{x} h_{1}\left(u_{i}, v_{j}\right)+R_{1}\left(u, v, u_{1}, v_{1}\right)=D_{x} \theta_{1}, \\
& D_{t} \tilde{\rho}_{1}=D_{x} \tilde{h}_{1}\left(u_{i}, v_{j}\right)+\tilde{R}_{1}\left(u, v, u_{1}, v_{1}\right)=D_{x} \tilde{\theta}_{1} .
\end{aligned}
$$

Therefore, we have set $\theta_{1}=h_{1}\left(u_{i}, v_{j}\right)+q_{1}(u, v), \tilde{\theta}_{1}=\tilde{h}_{1}\left(u_{i}, v_{j}\right)+\tilde{q}_{1}(u, v)$, where $q_{1}$ and $\tilde{q}_{1}$ are unknown functions and $D_{x} q_{1}=R_{1}, D_{x} \tilde{q}_{1}=\tilde{R}_{1}$. This trick allows us to evaluate $\rho_{4}, \tau_{4}, \tilde{\rho}_{4}, \tilde{\tau}_{4}$ and verify the fourth integrability conditions (2.18). These conditions imply $f_{3}^{\prime \prime}=g_{3}^{\prime \prime}=0$, hence

$$
f_{3}=a_{1} v+a_{2}, \quad g_{3}=a_{3} u+a_{4} .
$$

To simplify the further analysis one must list all irreducible cases of $f_{1}$ (or $g_{2}$ ). Let us take $f_{1}=c_{1} u^{2}+c_{2} u+c_{3} v^{2}+c_{4} v+c_{5}$ for definiteness.

Lemma 4. Using complex dilatations of $u$ and $v$, translations $u \rightarrow u+\lambda_{1}, v \rightarrow v+\lambda_{2}$ and the Galilei transformation $u_{t} \rightarrow u_{t}+\alpha u_{x}, v_{t} \rightarrow v_{t}+\alpha v_{x}$ one can reduce $f_{1}$ to one of the following forms:
1) $u^{2}+v^{2}$
2) $u^{2}+\alpha v$;
3) $v^{2}+\alpha u$;
4) $u+v$;
5) $u$;
6) $v$; 7) $f_{1}=0$,

where $\alpha$ is any constant. Moreover, in the cases $4-7$ the function $g_{2}$ must be linear $\left(b_{1}=b_{3}=0\right)$ because otherwise the permutation $u \leftrightarrow v$ gives one of the cases 1-3.

In cases 1 and 3 contradictions follow from the integrability conditions (1.5) with $n=1,3$ and (2.18) with $n=2,4$. In case 2 the integrability conditions (1.5) with $n=1,3,5$ and (2.18) with $n=2,4$ are satisfied iff system (3.44) is reduced to a pair of independent equations. Thus, a nontrivial integrable system (3.41) must belong to the following class:

$$
\begin{aligned}
& u_{t}=u_{3}+\left(c_{2} u+c_{4} v\right) u_{1}+\left(u\left(a_{1} v+a_{2}\right)+f_{4}(v)\right) v_{1}, \\
& v_{t}=a v_{3}+\left(v\left(a_{3} u+a_{4}\right)+g_{4}(u)\right) u_{1}+\left(b_{2} u+b_{4} v+b_{5}\right) v_{1},
\end{aligned}
$$

and only the following cases are possible:

$$
\text { 4) } \left.\left.c_{2}=c_{4}=1 ; \quad 5\right) c_{2}=1, c_{4}=0 ; \quad 6\right) c_{2}=0, c_{4}=1 ; \text { 7) } c_{2}=c_{4}=0 \text {. }
$$

In case 4 the integrability conditions (1.5) with $n=1, \ldots, 5$ provide the functions $g_{4}=k_{1} u+k_{2}$, $f_{4}=k_{3} v+k_{4}$, the coefficients $a_{3}=0, a_{4}=-1+a_{2}+b_{2}, b_{4}=b_{2}=(a+1) a_{2}-2 a-1$ and the following equations:

$$
(a+1)\left(2 a_{2}+a a_{2}-2 a-1\right)=0, \quad a^{2}\left(a_{2}-2\right)-a\left(4 a_{2}^{2}-5-7 a_{2}\right)+16 a_{2}-14 a_{2}^{2}-3=0,
$$




$$
\begin{aligned}
& a^{5}\left(a_{2}-2\right)-a^{4}\left(4 a_{2}^{2}-7 a_{2}-6\right)-2 a^{3}\left(16 a_{2}^{2}-5 a_{2}-37\right) \\
& \quad-a^{2}\left(177 a_{2}^{2}-224 a_{2}-41\right)-a\left(236 a_{2}^{2}-353 a_{2}+102\right)-a_{2}\left(37 a_{2}-53\right)-17=0 .
\end{aligned}
$$

Using the package Groebner in Maple, one can obtain $a_{2}=(1-a) / 3, a^{2}+7 a+1=0$ or $a=(3 c-7) / 2, c^{2}=5$. Then, the remaining coefficients are also determined and we obtain

$$
\begin{aligned}
& u_{t}=u_{3}+(u+v) u_{1}+\frac{1}{2}((3-c) u+(5 c-11) v) v_{1}, \quad c^{2}=5, \\
& v_{t}=\frac{1}{2} v_{3}(3 c-7)+((c+2) u-v) u_{1}+\frac{1}{2}(c-3)(u+v) v_{1} .
\end{aligned}
$$

The following substitution

$$
V=\frac{1}{6}(c+1)(v-u), \quad U=\frac{1}{12}(c+3) u+\frac{1}{6} v
$$

reduces system (3.46) to the third Drinfeld-Sokolov system

$$
\begin{aligned}
& U_{t}=-8 U_{3}+3 V_{3}+6(V-8 U) U_{1}+12 U V_{1}, \\
& V_{t}=12 U_{3}-2 V_{3}+48 V U_{1}+12(2 U-V) V_{1}
\end{aligned}
$$

that has been presented first in [3]. Scaling

$$
t \rightarrow-\frac{1}{2} t, \quad U=\rightarrow \frac{1}{6} U, \quad V=\rightarrow-\frac{1}{3} V
$$

gives more symmetric form of system (3.46a)

$$
\begin{aligned}
& U_{t}=4 U_{3}+3 V_{3}+(4 U+V) U_{1}+2 U V_{1}, \\
& V_{t}=3 U_{3}+V_{3}-4 V U_{1}-2(V+U) V_{1}
\end{aligned}
$$

that was found in [14].

In case 5 the equations $a_{1}=a_{2}=0, g_{4}^{\prime} f_{4}^{\prime}=0, g_{4}^{\prime \prime \prime}=0$ follow from the integrability conditions (1.5) with $n=1, \ldots, 5$. This implies $f_{4} \neq 0$ because otherwise the first equation of (3.45) will be independent. Hence, there are two branches (1) $f_{4}=1$ and (2) $f_{4}^{\prime} \neq 0, g_{4}^{\prime}=0$. Along the first branch, if one use additionally the integrability conditions (1.5) with $n=7$ and solves a large polynomial system for constants, one can obtain the following system:

$$
u_{t}=u_{3}+u u_{1}+v_{1}, \quad v_{t}=-2 v_{3}-u v_{1},
$$

that can be transformed to (1.3) by a scaling.

Along the second branch the integrability conditions (1.5) with $n=1, \ldots, 5$ provide the following system

$$
u_{t}=u_{3}+u u_{1}-v v_{1}, \quad v_{t}=-2 v_{3}-u v_{1}
$$

that is equivalent to (1.2).

Case 6 is symmetric to case 5 : one can obtain $f_{4}^{\prime \prime \prime}=0, g_{4}^{\prime \prime}=0, a_{3}=a_{4}=b_{2}=0$ from the integrability conditions (1.5) with $n=1, \ldots, 5$. Hence $g_{4} \neq 0$ and we have two branches $g_{4}=1$ or $g_{4}=u$. Using the additional integrability conditions (1.5) with $n=6,7$ one can obtain equations (1.2) and (1.3).

There are many branches in case 7 but all of them provide linear or triangular systems only.

As one can see, classification of integrable systems of the form (3.41) is a sufficiently laborious task. System (2.21) contains eight unknown functions depending on four variables, therefore classification of these systems is much more difficult. 


\section{Differential substitutions}

A differential substitution is a pair of equations

$$
u=f\left(U, V, U_{x}, V_{x}, \ldots, U_{n}, V_{n}\right), \quad v=g\left(U, V, U_{x}, V_{x}, \ldots, U_{n}, V_{n}\right),
$$

where $f$ and $g$ are some smooth functions.

Definition 1. If for any solution $(U, V)$ of a system $(\Sigma)$ formulas (4.1) provide a solution $(u, v)$ of system (1.1), then one says that system (1.1) admits substitution (4.1).

In all cases that we know, the new systems $(\Sigma)$ belong to the same class $(1.1)$

$$
U_{t}=U_{x x x}+P\left(U, V, U_{x}, V_{x}, U_{x x}, V_{x x}\right), \quad V_{t}=a V_{x x x}+Q\left(U, V, U_{x}, V_{x}, U_{x x}, V_{x x}\right),
$$

with some smooth functions $P$ and $Q$. There exist some group-theoretical explanation of this fact for $\mathrm{KdV}$ type equations [35]. Our attempts to introduce another parameter $a^{\prime} \neq a$ in $(S)$ had no success.

Substituting (4.1) into (1.1) one obtains the following equations

$$
\begin{aligned}
& \left.\left(D_{x}^{3} f+F\left(f, g, D_{x} f, D_{x} g, D_{x}^{2} f, D_{x}^{2} g\right)-\partial_{t} f\right)\right|_{S}=0 \\
& \left.\left(a D_{x}^{3} g+G\left(f, g, D_{x} f, D_{x} g, D_{x}^{2} f, D_{x}^{2} g\right)-\partial_{t} g\right)\right|_{S}=0 .
\end{aligned}
$$

It is obvious that transition to the manifold $(S)$ in (4.2) is equivalent to a replacement of $\partial_{t}$ by the evolutionary differentiation $D_{t}$ performed in accordance with $(S)$ :

$$
\begin{aligned}
& D_{t} f=D_{x}^{3} f+F\left(f, g, D_{x} f, D_{x} g, D_{x}^{2} f, D_{x}^{2} g\right), \\
& D_{t} g=a D_{x}^{3} g+G\left(f, g, D_{x} f, D_{x} g, D_{x}^{2} f, D_{x}^{2} g\right),
\end{aligned}
$$

where

$$
D_{t} f=\sum_{i=1}^{n} \frac{\partial f}{\partial U_{i}} D_{x}^{i}\left(U_{3}+P\right)+\sum_{i=1}^{n} \frac{\partial f}{\partial V_{i}} D_{x}^{i}\left(a V_{3}+Q\right) .
$$

Another way to obtain (4.3) is to differentiate equations (4.1) with respect to $t$ in accordance with (1.1) and $(S)$ and exclude $u$ and $v$ by using (4.1). This algorithm and many others are coded in Maple, see for example [36].

To find the admissible functions $f, g, P, Q$ from (4.3) one can use the following easily provable formula:

$$
\frac{\partial}{\partial U_{k}} D_{x}^{m} f=\sum_{s=0}^{m}\left(\begin{array}{c}
m \\
s
\end{array}\right) D_{x}^{m-s} \frac{\partial f}{\partial U_{k-s}}, \quad \frac{\partial f}{\partial U_{-i}} \equiv 0 \quad \text { for } \quad i>0
$$

and the analogous formula for $\partial / \partial V_{k}$. Differentiating (4.3) with respect to $U_{n+3}$ and $V_{n+3}$, one obtains

$$
\frac{\partial f}{\partial V_{n}}=0, \quad \frac{\partial g}{\partial U_{n}}=0
$$

Other corollaries of (4.3) are too cumbersome to consider them in the general form.

Let us consider, as an example, the first order differential substitutions for system (1.2)

$$
u_{t}=u_{3}+v u_{1}, \quad v_{t}=-\frac{1}{2} v_{3}+u u_{1}-v v_{1} .
$$


According to (4.4) one has $f=f\left(U, V, U_{1}\right), g=g\left(U, V, V_{1}\right)$, hence equations (4.3) now read

$$
\begin{aligned}
& D_{x}^{3} f-f_{U}\left(U_{3}+P\right)-f_{V}\left(Q-V_{3} / 2\right)-f_{U_{1}}\left(U_{4}+D_{x} P\right)+g D_{x} f=0, \\
& g_{U}\left(U_{3}+P\right)+g_{V}\left(Q-V_{3} / 2\right)+g_{V_{1}}\left(D_{x} Q-V_{4} / 2\right)+\frac{1}{2} D_{x}^{3} g-f D_{x} f+g D_{x} g=0 .
\end{aligned}
$$

Differentiating (4.6) with respect to $U_{3}$ and $V_{3}$ one can obtain four equations:

$$
\begin{array}{rlrl}
\frac{\partial f}{\partial U_{1}} \frac{\partial P}{\partial U_{2}} & =3 D_{x} \frac{\partial f}{\partial U_{1}}, & \frac{\partial f}{\partial U_{1}} \frac{\partial P}{\partial V_{2}} & =\frac{3}{2} \frac{\partial f}{\partial V}, \\
\frac{\partial g}{\partial V_{1}} \frac{\partial Q}{\partial U_{2}} & =-\frac{3}{2} \frac{\partial g}{\partial U}, & \frac{\partial g}{\partial V_{1}} \frac{\partial Q}{\partial V_{2}}=-\frac{3}{2} D_{x} \frac{\partial g}{\partial V_{1}} .
\end{array}
$$

Let us consider some corollaries of these equations.

1. If $\partial f / \partial U_{1}=0$ and $\partial g / \partial V_{1}=0$, then $u=f(U), v=g(V)$ is a trivial point transformation.

2. If $\partial f / \partial U_{1}=0$, then $u=f(U)$ and one can set $f(U)=U$ by modulo of the point transformation. In this case $P=g U_{1}$ from the first of equations (4.6).

3. If $\partial g / \partial V_{1}=0$, then $v=g(V)$ and one can set $g(V)=V$ by modulo of the point transformation. In this case $Q=f D_{x} f-V V_{1}$ from the second of equations (4.6).

4. If $\left(\partial f / \partial U_{1}\right)\left(\partial g / \partial V_{1}\right) \neq 0$, then one can find $P$ and $Q$ as polynomials of $U_{2}$ and $V_{2}$ from equations (4.7).

Investigation of cases 2-4 provides seven nontrivial solutions of equations (4.6) (see below $(3.1) \rightarrow(3.2), \ldots,(3.1) \rightarrow(3.17))$.

Note that integrable system (3.6) admits strange differential substitutions that generate nonintegrable systems. For example, system (3.6) admits the following differential substitution:

$$
\begin{aligned}
& u=\frac{3}{2} V_{2}-\frac{3}{4} V_{1}^{2}-\frac{3}{2} U_{1} e^{V}, \\
& v=\frac{9}{4}\left(-V_{4}+V_{1} V_{3}+V_{1}^{2} V_{2}-\frac{1}{4} V_{1}^{4}-U_{1}^{2} e^{2 V}+e^{V}\left(U_{3}+2 U_{2} V_{1}+3 U_{1} V_{2}\right)\right),
\end{aligned}
$$

so that the functions $U$ and $V$ satisfy the following system:

$$
\begin{aligned}
& U_{t}=U_{3}+\frac{3}{2} U_{2} V_{1}+\frac{3}{4} U_{1} V_{1}^{2}-U_{1} e^{-V} f^{\prime \prime}(U)+f(U), \\
& V_{t}=-\frac{1}{2} V_{3}+\frac{1}{4} V_{1}^{3}+\frac{3}{2} e^{V}\left(U_{2}+U_{1} V_{1}\right)-f^{\prime}(U)
\end{aligned}
$$

with arbitrary function $f$. This system does not satisfy the integrability conditions (1.4). To comprehend this unusual phenomenon we evaluate $V_{2}, V_{3}$ and $V_{4}$ from the first equation (4.8)

$$
\begin{aligned}
& V_{2}=\frac{2}{3} u+U_{1} e^{V}+\frac{1}{2} V_{1}^{2}, \quad V_{3}=\frac{2}{3} u_{1}+D_{x}\left(U_{1} e^{V}\right)+V_{1} V_{2}, \\
& V_{4}=\frac{2}{3} u_{2}+D_{x}^{2}\left(U_{1} e^{V}\right)+D_{x}\left(V_{1} V_{2}\right),
\end{aligned}
$$

and substitute them into the second one. The result is

$$
v=-u^{2}-\frac{3}{2} u_{2}
$$

It is easily verified that the obtained constraint is a reduction of system (3.6) into the single $\mathrm{KdV}$ equation $u_{t}=-1 / 2 u_{3}-u u_{1}$. This means that using a substitution like (4.8) we are trying to construct an integrable system from the single KdV equation. There are other such examples for system (3.6). Note that the reduction obtained above follows from the reduction $u=$ const for system (3.3) (see (3.3) and (3.3) $\rightarrow(3.6)$ ). 
To organize the presented list of systems we have computed admissible differential substitutions for each system and present the results in this section. The formula

$$
u^{\prime}=f\left(u, v, u_{x}, v_{x}, \ldots\right), \quad v^{\prime}=g\left(u, v, u_{x}, v_{x}, \ldots\right)
$$

will denote that if $u^{\prime}$ and $v^{\prime}$ are substituted into system (A), then system (B) follows for $u$ and $v$. We say in this case that system (B) is obtained from system (A) by the differential substitution.

Substitution $(\mathrm{A}) \rightarrow(\mathrm{B})$ establishes an interrelation between the sets of solutions of systems (A) and (B): $(u, v) \mapsto\left(u^{\prime}, v^{\prime}\right)$ is a single valued map. And conversely, if for some solution $\left(u^{\prime}, v^{\prime}\right)$ of system $(\mathrm{A})$ one solves the system of two ordinary differential equations $(\mathrm{A}) \rightarrow(\mathrm{B})$ for $u$ and $v$, then one or more solutions of system (B) are obtained. Of course, explicit solutions can be obtained very rarely when the substitution is linear or invertible (see below).

Let us consider the following simple example:

$$
u^{\prime}=u_{1}, \quad v^{\prime}=v_{1} .
$$

This substitution is possible for any divergent system

$$
u_{t}=\left(u_{2}+F\left(u, v, u_{1}, v_{1}\right)\right)_{x}, \quad v_{t}=\left(-\frac{1}{2} v_{2}+G\left(u, v, u_{1}, v_{1}\right)\right)_{x} .
$$

It produces the system $u_{t}=u_{3}+F\left(u_{1}, v_{1}, u_{2}, v_{2}\right), v_{t}=-v_{2} / 2+G\left(u_{1}, v_{1}, u_{2}, v_{2}\right)$ without $u_{0}$ and $v_{0}$. The inverse transformation is quasi-local $u=D_{x}^{-1} u^{\prime}, v=D_{x}^{-1} v^{\prime}$. This is a well known fact, that is why the substitutions $(u, v) \rightarrow\left(u_{1}, v_{1}\right)$ are not written for the divergent systems below. In some cases analogous substitutions are not so obvious and we present them likewise $(3.1) \rightarrow(3.2)$, for example.

Theorem 1. Differential substitutions presented below connect all systems from the list of Section 3 with systems (1.2) and (1.3). Systems (1.2) and (1.3) are also implicitly connected with each other.

The proof can be obtained by a direct verification.

List of the substitutions:

$$
\begin{aligned}
& u^{\prime}=u, \quad v^{\prime}=v_{1} ; \\
& u^{\prime}=\frac{3}{\sqrt{2}}\left(u_{2}-u_{1} v_{1}\right), \quad v^{\prime}=\frac{3}{2} v_{2}-\frac{3}{4}\left(u_{1}^{2}+v_{1}^{2}\right) ; \\
& u^{\prime}=\frac{3}{\sqrt{2}}\left(u_{1}-u v\right), \quad v^{\prime}=\frac{3}{2} v_{1}-\frac{3}{4}\left(u^{2}+v^{2}\right) ; \\
& u^{\prime}=\frac{3}{4} \sqrt{2}\left(u_{1}^{2}-2 u_{2}+2 v_{1}\right), \quad v^{\prime}=\frac{3}{2} v_{1} ; \\
& u^{\prime}=\frac{3}{4} \sqrt{2}\left(u^{2}-2 u_{1}+2 v\right), \quad v^{\prime}=\frac{3}{2} v ; \\
& u^{\prime}=3 \sqrt{2}\left((u+v)^{2}-u_{1}\right)+\frac{3}{16} \sqrt{2} c_{1}^{2}, \quad v^{\prime}=3 v_{1}+\frac{3}{2} c_{1}(u+v) ; \\
& u^{\prime}=\sqrt{2} u, \quad v^{\prime}=-3 v_{1}+u-3 v^{2} ; \\
& u^{\prime}=u, \quad v^{\prime}=k+\sqrt{u^{2}+v_{1}} ; \\
& u^{\prime}=\sqrt{v^{2}+u_{1}}, \quad v^{\prime}=v-k ; \\
& u^{\prime}=\sqrt{2}\left(\frac{4}{3} u+\frac{3}{16} c_{1}^{2}\right), \quad u_{1}+v_{2} \\
& u^{\prime}=\frac{3}{2} \sqrt{2}\left(\frac{1}{2} u_{1}^{2}-u_{2}+v_{1}+c_{1} e^{u}+2 c_{2} e^{2 u}\right), \quad c_{1} \sqrt{u+v_{1}} ; \\
& \left.u^{\prime}=\sqrt{2} u, \quad v^{\prime}=-\frac{3}{2} v_{2}-\frac{3}{4} v_{1}^{2}+u-c_{1}+c_{1} e^{u}\right) ; \\
& u^{\prime}=2 c_{1} u, \quad v^{\prime}=-\frac{3}{2} v_{2}-\frac{3}{4} v_{1}^{2}-\frac{1}{3} u^{2} e^{2 v}-\frac{3}{2} c_{1}^{2} e^{-2 v}+e^{v}\left(u_{1}+2 u v_{1}\right) ; \\
& u^{\prime}=\sqrt{3} u_{1} e^{v}+2 c_{1} u, \quad
\end{aligned}
$$




$$
\begin{aligned}
& v^{\prime}=\frac{3}{2} v_{2}-\frac{3}{4} v_{1}^{2}+2 \sqrt{3} c_{1} v_{1} e^{-v}-\frac{1}{2} e^{2 v}\left(u^{2}+c_{2}\right)-c_{1}^{2} e^{-2 v} ; \\
& u^{\prime}=\frac{1}{3} \sqrt{2} e^{v}\left(3 u_{1}+c_{1} e^{v}+2 u^{2} e^{v}\right), \\
& v^{\prime}=\frac{3}{2} v_{2}-\frac{3}{4} v_{1}^{2}-u_{1} e^{v}+\frac{1}{3} e^{2 v}\left(c_{1}-2 u^{2}\right) ; \\
& u^{\prime}=\frac{3}{2} \sqrt{2} e^{v}\left(u_{1}+2 c_{2} u+c_{1} u^{2} e^{v}\right), \\
& v^{\prime}=\frac{3}{2} v_{2}-\frac{3}{4} v_{1}^{2}-\frac{3}{2} c_{1} u_{1} e^{v}+3 c_{2} v_{1}-\frac{3}{4}\left(2 c_{2}+c_{1} u e^{v}\right)^{2}-\frac{3}{4} u^{2} e^{2 v} ; \\
& u^{\prime}=\frac{3}{2} \sqrt{2} e^{v}\left(u_{1}+\left(c+u^{2}\right) e^{v}\right), \quad v^{\prime}=\frac{3}{2} v_{2}-\frac{3}{4} v_{1}^{2}-\frac{3}{2} u_{1} e^{v}+\frac{3}{2}\left(c-u^{2}\right) e^{2 v} ; \\
& u^{\prime}=\frac{3}{2} \sqrt{2}\left(u_{2}-u_{1} v_{1}\right)+\sqrt{2}\left(c_{2} e^{u}-c_{3} e^{-u}-3 c_{1} u_{1} e^{-v}\right), \\
& v^{\prime}=\frac{3}{2} v_{2}-\frac{3}{4}\left(u_{1}^{2}+v_{1}^{2}\right)+c_{2} e^{u}+c_{3} e^{-u}-6 c_{1} v_{1} e^{-v}-3 c_{1}^{2} e^{-2 v} ; \\
& u^{\prime}=\frac{3}{2} \sqrt{2}\left(u_{2}-u_{1} v_{1}\right)-3 \sqrt{2}\left(c u_{1}+2 k v_{1}\right) e^{-v}-6 c k \sqrt{2} e^{-2 v}, \\
& v^{\prime}=\frac{3}{2} v_{2}-\frac{3}{4}\left(u_{1}^{2}+v_{1}^{2}\right)-3\left(k u_{1}+2 c v_{1}\right) e^{-v}-3\left(c^{2}+k^{2}\right) e^{-2 v} ; \\
& u^{\prime}=\frac{3}{\sqrt{2}}\left(u_{2}-u_{1} v_{1}\right)-\sqrt{2}\left(c_{2} e^{-u}+6 c_{1} c_{3} e^{u}-3 c_{1} u_{1} e^{u+v}+3 c_{3} u_{1} e^{-v}\right), \\
& v^{\prime}=\frac{3}{2} v_{2}-\frac{3}{4}\left(u_{1}^{2}+v_{1}^{2}\right)+c_{2} e^{-u}-3 c_{1}^{2} e^{2(u+v)}-3 c_{1} u_{1} e^{u+v}-6 c_{3} v_{1} e^{-v}-3 c_{3}^{2} e^{-2 v} \text {; } \\
& u^{\prime}=\frac{3}{\sqrt{2}}\left(u_{2}-u_{1} v_{1}\right)+3 \sqrt{2}\left(c_{1} e^{2(u+v)}+c_{2} u_{1} e^{u+v}\right), \\
& v^{\prime}=\frac{3}{2} v_{2}-\frac{3}{4}\left(u_{1}^{2}+v_{1}^{2}\right)+3\left(c_{1}-c_{2}^{2}\right) e^{2(u+v)}-3 c_{2} u_{1} e^{u+v} ; \\
& u^{\prime}=\sqrt{2}\left(\frac{3}{2}\left(u_{2}-u_{1} v_{1}\right)-\frac{2}{3} k c_{1}^{2} e^{-2 v}+3 c_{2} u_{1} e^{u+v}+2 k c_{1} c_{2} e^{u}\right) \\
& +c_{1} \sqrt{2}\left(k u_{1}+2 v_{1}\right) e^{-v}, \quad k^{2}=1 \text {, } \\
& v^{\prime}=\frac{3}{2} v_{2}-\frac{3}{4}\left(u_{1}^{2}+v_{1}^{2}\right)-\frac{2}{3} c_{1}^{2} e^{-2 v}-3 c_{2}^{2} e^{2(u+v)}-3 c_{2} u_{1} e^{u+v} \\
& +2 c_{1} c_{2} e^{u}+c_{1}\left(u_{1}+2 k v_{1}\right) e^{-v} \text {; } \\
& u^{\prime}=\frac{3}{2} \sqrt{2}\left(u_{2}-u_{1} v_{1}\right)+3 \sqrt{2}\left(c_{1} e^{u+v}+c_{2} e^{v-u}-c_{3} e^{-v}\right) u_{1} \\
& +6 c_{3} \sqrt{2}\left(c_{2} e^{-u}-c_{1} e^{u}\right), \\
& v^{\prime}=\frac{3}{2} v_{2}-\frac{3}{4}\left(u_{1}^{2}+v_{1}^{2}\right)-3 c_{1}^{2} e^{2(u+v)}-3 c_{2}^{2} e^{2(v-u)} \\
& +3 u_{1}\left(c_{2} e^{v-u}-c_{1} e^{u+v}\right)-6 c_{3} v_{1} e^{-v}-6 c_{1} c_{2} e^{2 v}-3 c_{3}^{2} e^{-2 v} \text {; } \\
& u^{\prime}=\frac{3}{2} \sqrt{2}\left(u_{2}-u_{1} v_{1}\right)+\frac{1}{3} \sqrt{2} c_{2}^{2} e^{-2 v}-\frac{1}{2} \sqrt{2} c_{2}\left(u_{1}-4 v_{1}\right) e^{-v} \\
& -2 \sqrt{2} c_{1} c_{2} e^{u}+\frac{3}{2} \sqrt{2} c_{1}\left(2 c_{1} e^{2(u+v)}+3 u_{1} e^{u+v}\right), \\
& v^{\prime}=\frac{3}{2} v_{2}-\frac{3}{4}\left(u_{1}^{2}+v_{1}^{2}\right)-\frac{15}{4} c_{1}^{2} e^{2(u+v)}-\frac{9}{2} c_{1} u_{1} e^{u+v}+\frac{5}{2} c_{1} c_{2} e^{u} \\
& -\frac{5}{12} c_{2}^{2} e^{-2 v}+c_{2}\left(u_{1}-v_{1}\right) e^{-v} \text {; } \\
& u^{\prime}=\frac{3}{2} \sqrt{2}\left(u_{2}-u_{1} v_{1}\right)+3 \sqrt{2} u_{1}\left(c_{1} e^{u+v}+c_{2} e^{v-u}\right)-\frac{2}{3} \sqrt{2} c_{3}^{2} e^{-2 v} \\
& +2 \sqrt{2} c_{3}\left(c_{2} e^{-u}-c_{1} e^{u}\right)-\sqrt{2} c_{3}\left(u_{1}+2 v_{1}\right) e^{-v}, \\
& v^{\prime}=\frac{3}{2} v_{2}-\frac{3}{4}\left(u_{1}^{2}+v_{1}^{2}\right)-3 e^{2 v}\left(c_{1} e^{u}+c_{2} e^{-u}\right)^{2}-c_{3}\left(u_{1}+2 v_{1}\right) e^{-v} \\
& +2 c_{3}\left(c_{2} e^{-u}-c_{1} e^{u}\right)-\frac{2}{3} c_{3}^{2} e^{-2 v}+3 u_{1}\left(c_{2} e^{v-u}-c_{1} e^{u+v}\right) \text {; } \\
& u^{\prime}=\frac{3}{\sqrt{2}} \frac{u_{2}-3 g v_{1}-2 g^{3}}{\sqrt{u_{1}-g^{2}}}-3 \sqrt{2}(3 g+2 c) \sqrt{u_{1}-g^{2}}, \\
& v^{\prime}=3\left(v_{1}-2 c g-c^{2}\right), \quad g=u+v \text {; } \\
& u^{\prime}=u, \quad v^{\prime}=v_{1} \text {; } \\
& u^{\prime}=\frac{3}{2} u_{2}+u^{2}+v, \quad v^{\prime}=u,
\end{aligned}
$$




$$
\begin{aligned}
& u^{\prime}=4 u_{2}+u_{1}\left(\frac{16}{3} \sqrt{u+v_{1}}-2 c_{1}\right)+\frac{16}{9} u^{2}+\frac{1}{2} c_{1}^{2} u, \\
& v^{\prime}=-\frac{u_{1}+v_{2}}{\sqrt{u+v_{1}}}-\frac{4}{3} v_{1}+c_{1} \sqrt{u+v_{1}} \\
& u^{\prime}=u_{1}, \quad v^{\prime}=v ; \\
& u^{\prime}=u_{1}, \quad v^{\prime}=v_{1} ; \\
& u^{\prime}=\frac{3}{2} u_{3}+2 u u_{1}+v_{1}, \quad v^{\prime}=u,
\end{aligned}
$$

this substitution has the quasi-local inverse substitution, see below (3.6) $\rightarrow(3.4)$;

$u^{\prime}=u_{2}+v v_{1}, \quad v^{\prime}=v-k ;$

$u^{\prime}=3 u_{3}-6\left(u_{1} v_{1}+v u_{2}\right)+2 u u_{1}, \quad v^{\prime}=3 v_{1}-3 v^{2}+u ;$

$u^{\prime}=e^{-v}, \quad v^{\prime}=\frac{3}{2} v_{2}-\frac{3}{4} v_{1}^{2}-u e^{2 v} ;$

$u^{\prime}=u_{1}+\sqrt{3} e^{v}\left(u_{2}+u_{1} v_{1}\right)$,

$v^{\prime}=\frac{3}{2} v_{2}-\frac{3}{4} v_{1}^{2}-u e^{2 v}+\sqrt{3} v_{1} e^{-v}-\frac{1}{4} e^{-2 v} ;$

$u^{\prime}=v, \quad v^{\prime}=-\frac{3}{2} v_{2}-v^{2}+u ;$

$u^{\prime}=v, \quad v^{\prime}=-\frac{3}{2} v_{2}-v^{2}+w, \quad w_{x}=u, \quad w_{t}=u_{2}+u v ;$

$u^{\prime}=v_{1}, \quad v^{\prime}=-\frac{3}{2} v_{3}-v_{1}^{2}+u$;

$u^{\prime}=u+k, \quad v^{\prime}=v_{1}-\frac{1}{2} v^{2}$;

$u^{\prime}=v-k, \quad v^{\prime}=u_{1}-\frac{3}{2} v_{2}-\frac{1}{2}\left(v^{2}+3 k^{2}\right)+2 k v ;$

$u^{\prime}=3 u_{1}-\frac{3}{2} v_{1}-\frac{3}{4}\left(u^{2}+v^{2}\right)$,

$v^{\prime}=\frac{9}{4} v_{3}-\frac{9}{4} u\left(u_{2}+2 v_{2}\right)+\frac{9}{4} v v_{2}-\frac{9}{2} u_{1}^{2}+\frac{9}{4} u^{2}\left(2 u_{1}+v_{1}\right)$

$$
-\frac{9}{4} v v_{1}(2 u+v)-\frac{9}{16}\left(u^{2}-v^{2}\right)^{2}
$$

$u^{\prime}=-3 u_{2}+\frac{3}{2} v_{1}$,

$v^{\prime}=\frac{9}{4} v_{3}+\frac{9}{2} u_{1} v_{2}-\frac{9}{16}\left(u_{1}^{2}-2 u_{2}+2 v_{1}\right)^{2}+\frac{9}{4}\left(u_{1}^{2}+v_{1}\right)^{2}-\frac{9}{8} u_{1}^{4} ;$

$u^{\prime}=-3 u_{1}+\frac{3}{2} v$,

$v^{\prime}=\frac{9}{4} v_{2}+\frac{9}{2} u v_{1}-\frac{9}{16}\left(u^{2}-2 u_{1}+2 v\right)^{2}+\frac{9}{4}\left(u^{2}+v\right)^{2}-\frac{9}{8} u^{4} ;$

$u^{\prime}=-3\left(v_{1}+2 u_{1}\right)+\frac{3}{2} c_{1} g, \quad g=u+v$,

$v^{\prime}=\frac{9}{2} v_{3}+\frac{9}{4} c_{1}\left(u_{2}-v_{2}\right)+18 g v_{2}-9 u_{1}^{2}+9 v_{1}^{2}$

$$
+9\left(c_{1} g+2 g^{2}\right) u_{1}-\frac{9}{8} c_{1}^{2}\left(u_{1}+g^{2}\right)+36 g^{2} v_{1}+9 g^{4} ;
$$

$u^{\prime}=3 v_{1}+u-3 v^{2}$,

$v^{\prime}=-\frac{9}{2} v_{3}+\frac{3}{2} u_{2}+9 v v_{2}-6 u_{1} v+6\left(3 v^{2}-u\right) v_{1}+3 v^{2}\left(2 u-3 v^{2}\right) ;$

$u^{\prime}=-\frac{u_{1}+v_{2}}{\sqrt{u+v_{1}}}+c_{1} \sqrt{u+v_{1}}-\frac{4}{3} v_{1}$,

$v^{\prime}=\frac{3}{2} \frac{u_{3}+v_{4}}{\sqrt{u+v_{1}}}-\frac{3}{4} c_{1} \frac{u_{2}+v_{3}}{\sqrt{u+v_{1}}}+\frac{8}{3} u \frac{u_{1}+v_{2}}{\sqrt{u+v_{1}}}+\frac{9}{8} \frac{\left(u_{1}+v_{2}\right)^{3}}{\left(u+v_{1}\right)^{5 / 2}}-\frac{\left(u_{1}+v_{2}\right)^{2}}{u+v_{1}}$

$$
\begin{aligned}
& -\frac{3}{8} \frac{u 1+v_{2}}{\left(u+v_{1}\right)^{3 / 2}}\left(6\left(u_{2}+v_{3}\right)-c_{1}\left(u_{1}+v_{2}\right)\right)+2\left(v_{3}+2 u_{2}+c_{1} v_{2}\right) \\
& +\frac{16}{9}\left(u^{2}-v_{1}^{2}\right)-\frac{8}{3}\left(v_{2}-u_{1}-c_{1} v_{1}\right) \sqrt{u+v_{1}}-\frac{1}{2} c_{1}^{2}\left(u+2 v_{1}\right) ;
\end{aligned}
$$

$u^{\prime}=\sqrt{u+v_{1}}-k$,

$v^{\prime}=-\frac{3}{4} \frac{u_{2}+v_{3}}{\sqrt{u+v_{1}}}+\frac{3}{8} \frac{\left(u_{1}+v_{2}\right)^{2}}{\left(u+v_{1}\right)^{3 / 2}}+2 k \sqrt{u+v_{1}}-v_{1}-\frac{u}{2} ;$

$u^{\prime}=i \sqrt{6} u_{1}+u^{2}+v, \quad v^{\prime}=\frac{3}{2}\left(u_{1}^{2}-v_{2}\right)+i \sqrt{6} u v_{1}-v^{2}$; 


$$
\begin{aligned}
& u^{\prime}=3\left(u_{1}+v_{1}-k u\right), \\
& v^{\prime}=-\frac{9}{2} v_{3}-\frac{9}{2} u u_{2}+9(u+k) v_{2}-\frac{27}{4} u_{1}^{2}-9 v_{1}^{2} \\
& +9 u(u+k) u_{1}-9\left(u^{2}+k^{2}\right) v_{1}-\frac{9}{4} u^{2}\left(u^{2}+2 k^{2}\right) ; \\
& u^{\prime}=-3 u_{2}+\frac{3}{2} v_{1}+\frac{3}{2} e^{u}\left(c_{1}+4 \sqrt{c_{2}} u_{1}\right), \\
& v^{\prime}=\frac{9}{4} v_{3}-\frac{9}{4}\left(\frac{1}{2} u_{1}^{2}-u_{2}+v_{1}+c_{1} e^{u}+2 c_{2} e^{2 u}\right)^{2}+\frac{9}{4} c_{1} u_{2} e^{u}+\frac{9}{2} u_{1} v_{2}+\frac{9}{4} v_{1}^{2} \\
& +\frac{9}{8} u_{1}^{2}\left(u_{1}^{2}+4 v_{1}\right)+\frac{9}{4} e^{u}\left(c_{1}\left(5 u_{1}^{2}+2 v_{1}\right)-4 \sqrt{c_{2}}\left(v_{2}+2 u_{1} v_{1}+u_{1}^{3}\right)\right)+18 c_{2}^{2} e^{4 u} \\
& +\frac{9}{4} e^{2 u}\left(c_{1}\left(c_{1}-12 \sqrt{c_{2}} u_{1}\right)+4 c_{2}\left(3 u_{1}^{2}+2 v_{1}\right)\right)+18 c_{2} e^{3 u}\left(c_{1}-2 \sqrt{c_{2}} u_{1}\right) \text {; } \\
& u^{\prime}=-3 u_{2}-\frac{3}{2} v_{2}-\frac{3}{4}\left(u_{1}^{2}+v_{1}^{2}\right)-2 \sqrt{6 c_{2}}\left(u_{1}+v_{1}\right) e^{u+v}-c_{1} e^{-2 v} \\
& -c_{2} e^{2(u+v)}+c_{3} e^{2(v-u)} \text {, } \\
& v^{\prime}=\frac{9}{4} v_{4}-\frac{9}{4} u_{1} u_{3}+\frac{9}{4} v_{3}\left(2 u_{1}+v_{1}\right)-\frac{9}{2} u_{2}\left(u_{2}+u_{1}^{2}\right)-\frac{9}{16}\left(u_{1}^{2}-v_{1}^{2}\right)^{2} \\
& +\frac{9}{4} v_{2}\left(u_{1}^{2}+2 u_{1} v_{1}-v_{1}^{2}\right)+4 \sqrt{6 c_{2}} v_{1}\left(2 c_{3} e^{3 v-u}-c_{1} e^{u-v}\right)+c \\
& +3 \sqrt{6 c_{2}}\left(v_{3}-u_{1} u_{2}+v_{2}\left(2 u_{1}+v_{1}\right)+2 u_{1} v_{1}\left(u_{1}+v_{1}\right)\right) e^{u+v} \\
& +6 v_{2}\left(c_{2} e^{2(u+v)}+c_{3} e^{2(v-u)}-c_{1} e^{-2 v}\right)+\frac{3}{2} c_{1}\left(u_{1}-v_{1}\right)\left(u_{1}-3 v_{1}\right) e^{-2 v} \\
& -c_{1}^{2} e^{-4 v}+4 c_{2} c_{3} e^{4 v}-\frac{3}{2} c_{2}\left(u_{1}-v_{1}\right)^{2} e^{2(u+v)}+\frac{3}{2} c_{3}\left(u_{1}-3 v_{1}\right)^{2} e^{2(v-u)} ; \\
& u^{\prime}=3 u_{2}-\frac{3}{2} v_{2}-\frac{3}{4}\left(u_{1}^{2}+v_{1}^{2}\right)-3 k e^{-v}\left(u_{1}+2 v_{1}\right)-3\left(c_{1}+2 k^{2}\right) e^{-2 v} \text {, } \\
& v^{\prime}=\frac{9}{4} v_{4}-\frac{9}{4} u_{1} u_{3}+\frac{9}{4} v_{3}\left(v_{1}-2 u_{1}\right)+\frac{9}{2} u_{2}\left(u_{1}^{2}-u_{2}\right)-\frac{9}{16}\left(u_{1}^{2}-v_{1}^{2}\right)^{2} \\
& +\frac{9}{4} v_{2}\left(u_{1}^{2}-2 u_{1} v_{1}-v_{1}^{2}\right)-9 c_{1}^{2} e^{-4 v}+18 k c_{1} e^{-3 v}\left(u_{1}+2 v_{1}\right) \\
& +\frac{9}{2} e^{-2 v}\left(4 k^{2} u_{2}-4 c_{1} v_{2}-2 k^{2}\left(u_{1}+v_{1}\right)^{2}+c_{1}\left(u_{1}+v_{1}\right)\left(u_{1}+3 v_{1}\right)\right) \\
& -\frac{9}{2} k e^{-v}\left(u_{3}+2 v_{3}-4 u_{2}\left(u_{1}+v_{1}\right)+v_{2}\left(2 v_{1}-3 u_{1}\right)+u_{1}^{2}\left(u_{1}+2 v_{1}\right)\right) \text {, } \\
& c_{1}=c^{2}-k^{2} \text {; } \\
& u^{\prime}=-3 u_{2}-\frac{3}{2} v_{2}-\frac{3}{4}\left(u_{1}^{2}+v_{1}^{2}\right)-3 c_{1}^{2} e^{2(u+v)}-3 c_{1}\left(3 u_{1}+2 v_{1}\right) e^{u+v} \\
& +c_{2} e^{-u}-k e^{-2 u}, \quad k=3 c_{3}^{2} \text {, } \\
& v^{\prime}=\frac{9}{4}\left(v_{4}-u_{1} u_{3}\right)+\frac{9}{4} v_{3}\left(2 u_{1}+v_{1}\right)-\frac{9}{2} u_{2}\left(u_{2}+u_{1}^{2}\right)+\frac{9}{4} v_{2}\left(u_{1}^{2}+2 u_{1} v_{1}-v_{1}^{2}\right) \\
& -\frac{9}{16}\left(u_{1}^{2}-v_{1}^{2}\right)^{2}-9 c_{1}^{4} e^{4(u+v)}-18 c_{1}^{3}\left(3 u_{1}+2 v_{1}\right) e^{3(u+v)}+6 c_{1}^{2} c_{2} e^{u+2 v} \\
& +6 k c_{1}^{2} e^{2 u}+2 k c_{2} e^{-u-2 v}+6 k c_{1}\left(u_{1}-2 v_{1}\right) e^{u-v}+\frac{3}{2} c_{2} e^{-u}\left(u_{2}+2 v_{2}+v_{1}^{2}\right) \\
& -\frac{9}{2} c_{1} e^{u+v}\left(u_{3}-2 v_{3}+u_{2}\left(9 u_{1}+4 v_{1}\right)-v_{2}\left(u_{1}+2 v_{1}\right)+4 u_{1}^{2}\left(u_{1}+v_{1}\right)\right) \\
& -\frac{3}{2} k e^{-2 v}\left(4 v_{2}+u_{1}\left(4 v_{1}-u_{1}\right)-3 v_{1}^{2}\right)-k^{2} e^{-4 v}+6 c_{1} c_{2} e^{v}\left(u_{1}+2 v_{1}\right) ; \\
& u^{\prime}=3 u_{2}-\frac{3}{2} v_{2}-\frac{3}{4}\left(u_{1}^{2}+v_{1}^{2}\right)+3\left(c_{1}-c_{2}^{2}\right) e^{2(u+v)}+3 c_{2}\left(u_{1}+2 v_{1}\right) e^{u+v}, \\
& v^{\prime}=\frac{9}{4} v_{4}-\frac{9}{4} u_{1} u_{3}+\frac{9}{4} v_{3}\left(v_{1}-2 u_{1}\right)+\frac{9}{2} u_{2}\left(u_{1}^{2}-u_{2}\right)-\frac{9}{16}\left(u_{1}^{2}-v_{1}^{2}\right)^{2} \\
& +\frac{9}{4} v_{2}\left(u_{1}^{2}-2 u_{1} v_{1}-v_{1}^{2}\right)-9 k c_{2}^{2} e^{4(u+v)}+18 k c_{2} e^{3(u+v)}\left(u_{1}+2 v_{1}\right) \\
& +\frac{9}{2} e^{2(u+v)}\left(2 c_{2}^{2} u_{2}+4 c_{1} v_{2}+c_{1}\left(u_{1}+3 v_{1}\right)^{2}-c_{2}^{2}\left(u_{1}^{2}+4 u_{1} v_{1}+5 v_{1}^{2}\right)\right) \\
& -\frac{9}{2} c_{2} e^{u+v}\left(u_{3}+2 v_{3}+u_{2}\left(u_{1}+4 v_{1}\right)+v_{2}\left(2 v_{1}-u_{1}\right)\right), \quad k=c_{2}^{2}-2 c_{1} ;(3.6) \rightarrow \\
& u^{\prime}=3 u_{2}-\frac{3}{2} v_{2}-\frac{3}{4}\left(u_{1}^{2}+v_{1}^{2}\right)-3 c_{2}^{2} e^{2(u+v)}+3 c_{2}\left(u_{1}+2 v_{1}\right) e^{u+v} \\
& +2 c_{1} c_{2} e^{u}+c_{1} e^{-v}\left(u_{1}+2 v_{1}\right)-\frac{2}{3} c_{1}^{2} e^{-2 v}, \\
& v^{\prime}=\frac{9}{4} v_{4}-\frac{9}{4} u_{1} u_{3}+\frac{9}{4} v_{3}\left(v_{1}-2 u_{1}\right)+\frac{9}{2} u_{2}\left(u_{1}^{2}-u_{2}\right)-\frac{9}{16}\left(u_{1}^{2}-v_{1}^{2}\right)^{2} \\
& +\frac{9}{4} v_{2}\left(u_{1}^{2}-2 u_{1} v_{1}-v_{1}^{2}\right)-9 c_{2}^{4} e^{4(u+v)}+18 c_{2}^{3} e^{3(u+v)}\left(u_{1}+2 v_{1}\right) \\
& +12 c_{1} c_{2}^{3} e^{3 u+2 v}+\frac{9}{2} c_{2}^{2} e^{2(u+v)}\left(2 u_{2}-u_{1}^{2}-4 u_{1} v_{1}-5 v_{1}^{2}\right) \\
& -4 c_{1}^{2} c_{2}^{2} e^{2 u}+c_{1}^{2} e^{-2 v}\left(2 u_{2}-\left(u_{1}+v_{1}\right)^{2}\right)
\end{aligned}
$$




$$
\begin{aligned}
& -\frac{9}{2} c_{2} e^{u+v}\left(u_{3}+2 v_{3}+u_{2}\left(u_{1}+4 v_{1}\right)+v_{2}\left(2 v_{1}-u_{1}\right)\right) \\
& -6 c_{1} c_{2}^{2} e^{2 u+v}\left(u_{1}+2 v_{1}\right)-3 c_{1} c_{2} e^{u}\left(3 u_{2}+2 v_{2}+2 u_{1} v_{1}+3 v_{1}^{2}\right) \\
& +\frac{3}{2} c_{1} e^{-v}\left(u_{3}+2 v_{3}-4 u_{2}\left(u_{1}+v_{1}\right)+v_{2}\left(2 v_{1}-3 u_{1}\right)+u_{1}^{2}\left(u_{1}+2 v_{1}\right)\right) \\
& u^{\prime}=3 u_{2}-\frac{3}{2} v_{2}-\frac{3}{4}\left(u_{1}^{2}+v_{1}^{2}\right)-\frac{15}{4} c_{1}^{2} e^{2(u+v)}+\frac{9}{2} c_{1}\left(u_{1}+2 v_{1}\right) e^{u+v} \\
& +\frac{5}{2} c_{1} c_{2} e^{u}+c_{2} e^{-v}\left(u_{1}+2 v_{1}\right)-\frac{5}{12} c_{2}^{2} e^{-2 v}, \\
& v^{\prime}=\frac{9}{4} v_{4}-\frac{9}{4} u_{1} u_{3}+\frac{9}{4} v_{3}\left(v_{1}-2 u_{1}\right)+\frac{9}{2} u_{2}\left(u_{1}^{2}-u_{2}\right)-\frac{9}{16}\left(u_{1}^{2}-v_{1}^{2}\right)^{2} \\
& +\frac{9}{4} v_{2}\left(u_{1}^{2}-2 u_{1} v_{1}-v_{1}^{2}\right)-\frac{81}{16} c_{1}^{4} e^{4(u+v)}+\frac{27}{4} c_{1}^{3} e^{3(u+v)}\left(u_{1}+2 v_{1}\right) \\
& +\frac{27}{4} c_{1}^{3} c_{2} e^{3 u+2 v}+\frac{9}{8} c_{1}^{2} e^{2(u+v)}\left(18 u_{2}+16 v_{2}-5 u_{1}^{2}-12 u_{1} v_{1}-9 v_{1}^{2}\right) \\
& -\frac{27}{8} c_{1}^{2} c_{2}^{2} e^{2 u}+\frac{3}{4} c_{1} c_{2}^{3} e^{u-2 v}-\frac{9}{4} c_{1} c_{2}^{2} e^{u-v}\left(u_{1}+2 v_{1}\right) \\
& -\frac{27}{4} c_{1} e^{u+v}\left(u_{3}+2 v_{3}+u_{2}\left(u_{1}+4 v_{1}\right)+v_{2}\left(2 v_{1}-u_{1}\right)\right) \\
& -\frac{3}{4} c_{1} c_{2} e^{u}\left(17 u_{2}+14 v_{2}+12 u_{1} v_{1}+19 v_{1}^{2}\right)+\frac{1}{2} c_{2}^{3} e^{-3 v}\left(u_{1}+2 v_{1}\right) \\
& +\frac{1}{8} c_{2}^{2} e^{-2 v}\left(16 u_{2}+12 v_{2}-11 u_{1}^{2}-28 u_{1} v_{1}-17 v_{1}^{2}\right)-\frac{1}{16} c_{2}^{4} e^{-4 v} \\
& +\frac{3}{2} c_{2} e^{-v}\left(u_{3}+2 v_{3}-4 u_{2}\left(u_{1}+v_{1}\right)+v_{2}\left(2 v_{1}-3 u_{1}\right)+u_{1}^{2}\left(u_{1}+2 v_{1}\right)\right) \\
& u^{\prime}=3 \frac{2 g^{3}-u_{2}+2 g v_{1}}{\sqrt{u_{1}-g^{2}}}+6 g \sqrt{u_{1}-g^{2}}-6 u_{1}-3 v_{1}-3 c(2 g+c), \quad g=u+v, \\
& v^{\prime}=\frac{9}{2} v_{3}+9(3 g+c) u_{2}+9(2 g+c) v_{2}-\frac{9}{4} \frac{\left(2 g^{3}-u_{2}+2 g v_{1}\right)^{2}}{u_{1}-g^{2}}+9\left(2 u_{1}+v_{1}\right)^{2} \\
& -18 u_{1}^{2}+18 \sqrt{u_{1}-g^{2}}\left(v_{2}+2(2 g+c)\left(v_{1}+g^{2}\right)\right)+36(2 g+c)\left(u_{1}-g^{2}\right)^{3 / 2} \\
& -9 g^{2} u_{1}-18\left((g+c)^{2}+2 g^{2}\right) v_{1}-36 c g\left((g+c)^{2}+g^{2}\right)-45 g^{4} \text {; } \\
& u^{\prime}=v-2 k, \quad v^{\prime}=u-\frac{3}{2} v_{1}+\frac{4}{3} k^{3} t ; \\
& u^{\prime}=\frac{3}{2} u_{1}+v-\frac{4}{3} k^{3} t, \quad v^{\prime}=u+2 k ; \\
& u^{\prime}=-\frac{1}{2} v, \quad v^{\prime}=\sqrt{u+v_{1}} \text {; } \\
& u^{\prime}=c_{1} \sqrt{2} e^{-v}, \quad v^{\prime}=-v_{1}+\frac{2}{3} u e^{v} \text {; } \\
& u^{\prime}=u e^{v}, \quad v^{\prime}=v_{1}-c_{1} u e^{v}-2 c_{2} ; \\
& u^{\prime}=(u+\sqrt{-c}) e^{v}, \quad v^{\prime}=v_{1}+(\sqrt{-c}-u) e^{v} ; \\
& u^{\prime}=u_{1}+2 k e^{-v}, \quad v^{\prime}=v_{1}-2 c e^{-v} \text {; } \\
& u^{\prime}=u_{1}+c e^{u+v}, \quad v^{\prime}=v_{1}-k e^{u+v}, \\
& u^{\prime}=\frac{3}{2}\left(v-u_{1}\right)+\frac{3}{4} u^{2}, \quad v^{\prime}=-\frac{1}{2} u ; \\
& u^{\prime}=\frac{3}{2}\left(u_{1}-u v\right), \quad v^{\prime}=\frac{1}{2}(u-v) \text {; } \\
& u^{\prime}=3(u+v)^{2}-3 u_{1}+\frac{3}{16} c_{1}^{2}, \quad v^{\prime}=\frac{1}{4} c_{1}-u-v ; \\
& u^{\prime}=u, \quad v^{\prime}=v_{1} \text {; } \\
& u^{\prime}=\frac{4}{3} u+\frac{3}{16} c_{1}^{2}, \quad v^{\prime}=\frac{1}{4} c_{1}-\frac{2}{3} \sqrt{u+v_{1}} ;
\end{aligned}
$$




$$
\begin{aligned}
& u^{\prime}=u, \quad v^{\prime}=\frac{1}{2} v_{1}+\frac{1}{\sqrt{3}} c e^{-v} ; \\
& u^{\prime}=c_{1} \sqrt{2} u, \quad v^{\prime}=\frac{1}{2} v_{1}-\frac{1}{3} u e^{v}-\frac{\sqrt{2}}{2} c_{1} e^{-v} ; \\
& u^{\prime}=-u_{1} e^{v}-\frac{1}{3} e^{2 v}\left(2 u^{2}+c_{1}\right), \quad v^{\prime}=-\frac{1}{2} v_{1}+\frac{1}{3} \sqrt{-2 c_{1}} e^{v} ; \\
& u^{\prime}=u_{1} e^{v}+\frac{1}{3} e^{2 v}\left(2 u^{2}+c_{1}\right), \quad v^{\prime}=-\frac{1}{2} v_{1}+\frac{2}{3} u e^{v} ; \\
& u^{\prime}=\frac{3}{2} e^{v}\left(u_{1}+2 c_{2} u+c_{1} u^{2} e^{v}\right), \quad v^{\prime}=-\frac{1}{2} v_{1}+\frac{1}{2}\left(c_{1}+1\right) u e^{v}+c_{2} ; \\
& u^{\prime}=\frac{9}{4}(u+v)^{2}-\frac{9}{4} u_{1}, \quad v^{\prime}=\frac{9}{4} u \\
& \text { this substitution is invertible: } \\
& u^{\prime}=\frac{4}{9} v, \quad v^{\prime}=\frac{2}{3} \sqrt{u+v_{1}}-\frac{4}{9} v .
\end{aligned}
$$

The graph of the substitutions is very cumbersome, therefore we show the most interesting subgraph only.

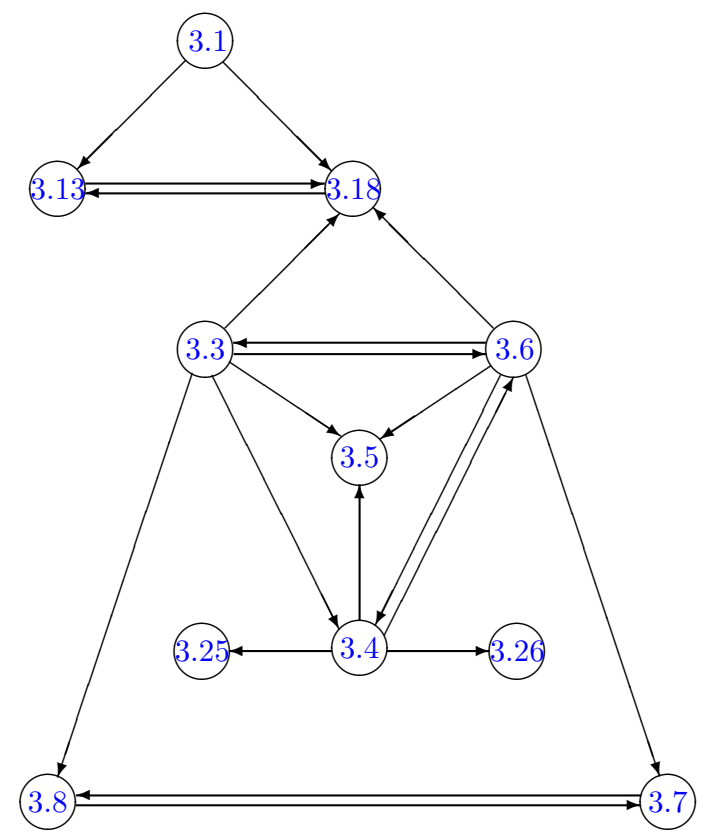

Fig. 1. A subgraph of the differential substitutions.

Comments. (1) System (3.1) coincides with (1.2) and (3.3) coincides with (1.3).

(2) It was simpler to obtain some systems from (3.6) than (3.3) or vice versa. These systems are connected by the second order invertible substitution, see $(3.6) \rightarrow(3.3)$ and $(3.3) \rightarrow(3.6)$. Hence, each system obtained from (3.6) can be obtained from (3.3) and vice versa. (3) Fifteen systems (3.9)-(3.15), (3.18), (3.22), (3.33)-(3.36), (3.38) and (3.40) can be obtained from both (3.1) and (3.6) by the presented differential substitutions. Twelve systems (3.2), (3.16), (3.17), (3.23), (3.24), (3.27)-(3.30), (3.32), (3.37) and (3.39) can be obtained from (3.1). The remaining eleven systems $(3.3)-(3.5),(3.7),(3.8),(3.19)-(3.21),(3.25),(3.26)$ and (3.31) can be obtained from (3.6) or (3.3).

Remark 7. As the systems (3.1) and (3.3) have the Lax representations, then all systems from the list have the Lax representations in a generalized meaning (see Section 5).

Remark 8. Some of the presented substitutions are superpositions of lower order substitutions, other substitutions are irreducible.

Remark 9. System (3.1) admits the first and second order substitutions and does not admit the third and fourth order substitutions. Probably it does not admit any higher order substitutions, 
either. Systems (3.4) and (3.6) admit the substitutions from the first till fourth orders. We do not present higher order substitutions for (3.4) because simpler substitutions exist for (3.6). Fifth and higher order substitutions for systems (3.4) and (3.6) have not been computed because the computations are extremely cumbersome.

Remark 10. There are some additional differential substitutions under the constraints for constants in the systems. For example, there are substitutions $(3.1) \rightarrow(3.31)$ for $c_{1}=0$ and $(3.6) \rightarrow(3.32)$ for $c_{3}=0$ or $(3.6) \rightarrow(3.39)$ for $c_{2}=0$ and so on. These substitutions are not so important and we do not present them here.

Remark 11. Unexpectedly, the well known systems (3.1) and (3.3) are implicitly connected as it is shown in Fig. 1.

\section{Examples of zero curvature representations}

The IST method for nonlinear equations with two independent variables is based on investigation of a linear overdetermined system

$$
L\left(\boldsymbol{u}, \lambda, \partial_{x}\right) \psi=0, \quad \psi_{t}=A\left(\boldsymbol{u}, \lambda, \partial_{x}\right) \psi,
$$

where $L$ and $A$ are ordinary linear operators, $\boldsymbol{u}$ is a smooth (vector) function satisfying a nonlinear partial differential equation $E(\boldsymbol{u})=0$ and $\lambda$ is a parameter. The operators $L, A$ may be both scalar and matrix. The operators $L, A$ and $E$ may be also pseudodifferential or integrodifferential. The compatibility condition of system (5.1) reads

$$
\left.\left(\frac{\partial L}{\partial t}+L A\right) \psi\right|_{L \psi=0}=0
$$

There are two ways to satisfy this condition. The first operator condition was introduced by P.D. Lax [37]:

$$
\frac{\partial L}{\partial t}+L A=A L, \quad \text { or } \quad \frac{\partial L}{\partial t}=[A, L] .
$$

The second more general operator condition was introduced in [38], see also [39]:

$$
\frac{\partial L}{\partial t}+L A=B L
$$

If an equation $E(\boldsymbol{u})=0$ is equivalent to equation (5.2), then (5.2) is said to be the Lax representation of the equation $E(\boldsymbol{u})=0$. The pair of operators $(L, A)$ is said to be the $(L, A)$ pair or the Lax pair.

If an equation $E(\boldsymbol{u})=0$ is equivalent to equation (5.3), then (5.3) is said to be the $(L, A, B)$ representation of the equation $E(\boldsymbol{u})=0$ or the triad representation.

In all cases, operator $L$ must essentially depend on the parameter $\lambda$. This parameter cannot be removed by a gauge transformation $L \rightarrow f^{-1} L f$ with some smooth function $f$, in particular.

If system (5.1) is differential, then the standard substitution $\psi=\Psi_{1}, \psi_{x}=\Psi_{2}$ and so on, provides the following first order system:

$$
\Psi_{x}=U \Psi, \quad \Psi_{t}=V \Psi,
$$

where $U$ and $V$ are square matrices depending on $\boldsymbol{u}$ and $\lambda$. The compatibility condition of linear system (5.4) reads

$$
U_{t}-V_{x}+[U, V]=0
$$


if $E(\boldsymbol{u})=0$. Usually a stronger condition is required: (5.5) is valid iff $E(\boldsymbol{u})=0$. In this case equation (5.5) is said to be the zero curvature representation. For an evolutionary system $\boldsymbol{u}_{t}=\boldsymbol{K}\left(\boldsymbol{u}, \boldsymbol{u}_{x}, \ldots, \boldsymbol{u}_{n}\right), \boldsymbol{u}=\left\{u^{\alpha}\right\}$ the matrix $U$ usually depend on $\boldsymbol{u}$ only, but it may depend on $\boldsymbol{u}, \boldsymbol{u}_{x}, \boldsymbol{u}_{x x}$, and so on. Let us consider the general case.

If some smooth functions $F=F\left(\boldsymbol{u}, \boldsymbol{u}_{x}, \ldots, \boldsymbol{u}_{r}\right)$ and $\Phi=\Phi\left(\boldsymbol{u}, \boldsymbol{u}_{x}, \ldots, \boldsymbol{u}_{p}\right)$ satisfy the condition

$$
\left.\left(\partial_{t} F+\Phi\right)\right|_{\boldsymbol{u}_{t}=\boldsymbol{K}}=0
$$

then one obtains

$$
\left.\Phi\right|_{\boldsymbol{u}_{t}=\boldsymbol{K}}=\Phi,\left.\quad \partial_{t} F\right|_{\boldsymbol{u}_{t}=\boldsymbol{K}}=\left.\frac{\partial F}{\partial u_{i}^{\alpha}} \partial_{t} u_{i}^{\alpha}\right|_{\boldsymbol{u}_{t}=\boldsymbol{K}}=\left.\frac{\partial F}{\partial u_{i}^{\alpha}} \partial_{x}^{i} u_{t}^{\alpha}\right|_{\boldsymbol{u}_{t}=\boldsymbol{K}}=\frac{\partial F}{\partial u_{i}^{\alpha}} D_{x}^{i} K^{\alpha},
$$

where the summation over $i=0, \ldots, r$ and $\alpha=1, \ldots, m$ is implied. This implies

$$
\Phi=-\frac{\partial F}{\partial u_{i}^{\alpha}} D_{x}^{i} K^{\alpha}
$$

according to (5.6). Using this result one obtains the following identity:

$$
\partial_{t} F+\Phi \equiv \frac{\partial F}{\partial u_{i}^{\alpha}} D_{x}^{i}\left(u_{t}^{\alpha}-K^{\alpha}\right), \quad \forall \boldsymbol{u}
$$

for any $F$ and $\Phi$ satisfying (5.6).

Let us apply identity (5.7) to equation (5.5). If the matrix $U$ depends on $\boldsymbol{u}$ only then

$$
U_{t}-V_{x}+[U, V]=\frac{\partial U}{\partial u^{\alpha}}\left(u_{t}^{\alpha}-K^{\alpha}\right), \quad \forall \boldsymbol{u} .
$$

It is obvious now that equation (5.5) is equivalent to $\boldsymbol{u}_{t}=\boldsymbol{K}$ iff the matrices $\partial U / \partial u^{\alpha}, \alpha=$ $1, \ldots, m$ are linearly independent. Suppose now that the matrix $U$ depends on $\boldsymbol{u}$ and $\boldsymbol{u}_{x}$ then one obtains

$$
U_{t}-V_{x}+[U, V]=\frac{\partial U}{\partial u^{\alpha}}\left(u_{t}^{\alpha}-K^{\alpha}\right)+\frac{\partial U}{\partial u_{x}^{\alpha}} D_{x}\left(u_{t}^{\alpha}-K^{\alpha}\right), \quad \forall \boldsymbol{u} .
$$

If the matrices $\partial U / \partial u^{\alpha}, \partial U / \partial u_{x}^{\beta}, \alpha, \beta=1, \ldots, m$ are linearly independent, then equation (5.5) is equivalent to $\boldsymbol{u}_{t}=\boldsymbol{K}$ again. Otherwise, equation (5.5) would be equivalent to some differential consequence of the system $\boldsymbol{u}_{t}=\boldsymbol{K}$ that is a more general system than the original one. In this case we call equation (5.5) the generalized zero curvature representation.

It is well known that equations (5.4) and (5.5) are covariant under the following transformation

$$
\bar{\Psi}=S^{-1} \Psi, \quad \bar{U}=S^{-1}\left(U S-S_{x}\right), \quad \bar{V}=S^{-1}\left(V S-S_{t}\right),
$$

where $S$ is any non-degenerate matrix. This transformation is called a gauge one. Any gauge transformation is invertible and preserves compatibility of system (5.4).

Two $(L, A)$-pairs were proposed for system $(1.2)$ in [3]. One of these $(L, A)$-pairs coincides with the $(L, A)$-pair that was presented in [4]. The $L$-operator of the common $(L, A)$-pair takes the form $L=\left(\partial_{x}^{2}+f\right)\left(\partial_{x}^{2}-g\right)$, where

$$
f=\frac{1}{6}(u \sqrt{2}-2 v), \quad g=\frac{1}{6}(u \sqrt{2}+2 v) .
$$


The temporal Lax equation reads $\psi_{t}=A \psi$, where $A$ is a fractional degree of $L$. The spatial Lax equation $L \psi=\lambda^{2} \psi$ can be transformed into the system $\left(\partial_{x}^{2}-g\right) \psi=\lambda \varphi,\left(\partial_{x}^{2}+f\right) \varphi=\lambda \psi$ and then into the normal form (5.4), were

$$
\begin{aligned}
U & =\left(\begin{array}{cccc}
0 & 1 & 0 & 0 \\
(u \sqrt{2}-2 v) / 6 & 0 & \lambda & 0 \\
0 & 0 & 0 & 1 \\
\lambda & 0 & -(u \sqrt{2}+2 v) / 6 & 0
\end{array}\right), \\
V & =\left(\begin{array}{cccc}
\left(u_{1} \sqrt{2}+v_{1}\right) / 6 & -\left(u_{1} \sqrt{2}+v_{1}\right) / 3 & 0 & -2 \lambda \\
f_{1}+f_{2} & -\left(u_{1} \sqrt{2}+v_{1}\right) / 6 & \lambda v / 3 & 0 \\
0 & -2 \lambda & -\left(u_{1} \sqrt{2}-v_{1}\right) / 6 & \left(u_{1} \sqrt{2}-v_{1}\right) / 3 \\
\lambda v / 3 & 0 & f_{1}-f_{2} & \left(u_{1} \sqrt{2}-v_{1}\right) / 6
\end{array}\right) .
\end{aligned}
$$

Here $f_{1}$ and $f_{2}$ take the following form:

$$
f_{1}=\frac{1}{18}\left(3 v_{2}-2 u^{2}+2 v^{2}\right)-2 \lambda^{2}, \quad f_{2}=\frac{\sqrt{2}}{18}\left(3 u_{2}+u v\right) .
$$

Matrices (5.11) realize the zero curvature representation of system (1.2).

System (1.3) also has two Lax representations (see [3]). Using the simpler $L$-operator, we have found, similarly to the previous case, the following matrices that realize the zero curvature representation of system (1.3):

$$
\begin{aligned}
\tilde{U} & =\left(\begin{array}{cccc}
0 & 1 & 0 & 0 \\
-v / 3-\lambda & 0 & 1 & 0 \\
0 & 0 & 0 & 1 \\
u / 9 & 0 & \lambda-v / 3 & 0
\end{array}\right), \\
\tilde{V} & =\left(\begin{array}{cccc}
v_{1} / 6 & 2 \lambda-v / 3 & 0 & -2 \\
h-\lambda v / 3 & -v_{1} / 6 & v / 3 & 0 \\
u_{1} / 9 & -2 u / 9 & v_{1} / 6 & -2 \lambda-v / 3 \\
u v / 27+u_{2} / 9 & -u_{1} / 9 & h+\lambda v / 3 & -v_{1} / 6
\end{array}\right),
\end{aligned}
$$

where

$$
h=\frac{1}{6} v_{2}+\frac{1}{9}\left(v^{2}-2 u\right)-2 \lambda^{2} .
$$

Let us consider an admissible differential substitution $u=f\left(\tilde{u}_{i}, \tilde{v}_{j}\right), v=g\left(\tilde{u}_{i}, \tilde{v}_{j}\right)$ of system (1.1). Substituting $u$ and $v$ in the matrices $U(u, v)$ and $V\left(u_{i}, v_{j}\right)$ one obtains

$$
\hat{U}\left(\tilde{u}_{i}, \tilde{v}_{j}\right)=U(f, g), \quad \hat{V}\left(\tilde{u}_{i}, \tilde{v}_{j}\right)=V\left(D_{x}^{k} f, D_{x}^{l} g\right) .
$$

As $\hat{U}$ depends on derivatives of $\tilde{u}$ or $\tilde{v}$, then one has a generalized zero curvature representation.

To obtain an ordinary zero curvature representation one can try to remove higher order derivatives from the matrix $\hat{U}$ using the gauge transformation (5.10). But this is not always possible (see example B below).

A. Performing the substitution $(3.1) \rightarrow(3.9)$ into the matrix $U$ from (5.11) one obtains

$$
\hat{U}=\left(\begin{array}{cccc}
0 & 1 & 0 & 0 \\
\left(u_{2}-v_{2}\right) / 2+h_{1} & 0 & \lambda & 0 \\
0 & 0 & 0 & 1 \\
\lambda & 0 & -\left(u_{2}+v_{2}\right) / 2+h_{2} & 0
\end{array}\right)
$$


where

$$
h_{1}=\frac{1}{4}\left(u_{1}-v_{1}\right)^{2}, \quad h_{2}=\frac{1}{4}\left(u_{1}+v_{1}\right)^{2} .
$$

One can easily verify that matrices $A=\hat{U}_{u_{2}}$ and $B=\hat{U}_{v_{2}}$ are commutative, hence the system $S_{x}=\left(A u_{x x}+B v_{x x}\right) S$ has the following solution $S=\exp \left(A u_{x}+B v_{x}\right)$. The matrix $U_{1}=\overline{\hat{U}}$ evaluated according to (5.10) takes the following form

$$
U_{1}=\frac{1}{2}\left(\begin{array}{cccc}
u_{x}-v_{x} & 2 & 0 & 0 \\
0 & v_{x}-u_{x} & 2 \lambda & 0 \\
0 & 0 & -u_{x}-v_{x} & 2 \\
2 \lambda & 0 & 0 & u_{x}+v_{x}
\end{array}\right)
$$

Now another gauge transformation is possible with the following diagonal matrix:

$$
S_{1}=\exp \left(\int \operatorname{diag}\left(U_{1}\right) d x\right)
$$

where $\operatorname{diag}\left(U_{1}\right)$ is the main diagonal of $U_{1}$. This gauge transformation provides the following matrix

$$
U_{2}=\left(\begin{array}{cccc}
0 & e^{v-u} & 0 & 0 \\
0 & 0 & \lambda e^{-v} & 0 \\
0 & 0 & 0 & e^{u+v} \\
\lambda e^{-v} & 0 & 0 & 0
\end{array}\right)
$$

A corresponding $V$-matrix can be obtained by solving equation (5.5) directly or by the previous twofold gauge transformation. This matrix takes the following form

$$
V_{2}=\left(\begin{array}{cccc}
0 & e^{v-u} f_{1} & \lambda e^{-u}\left(u_{1}+v_{1}\right) & -2 \lambda e^{v} \\
-2 \lambda^{2} e^{u-v} & 0 & \frac{\lambda}{4} e^{-v}\left(2 v_{2}-3 u_{1}^{2}+v_{1}^{2}\right) & \lambda e^{u}\left(u_{1}-v_{1}\right) \\
\lambda e^{u}\left(v_{1}-u_{1}\right) & -2 \lambda e^{v} & 0 & e^{u+v} f_{2} \\
\frac{\lambda}{4} e^{-v}\left(2 v_{2}-3 u_{1}^{2}+v_{1}^{2}\right) & -\lambda e^{-u}\left(u_{1}+v_{1}\right) & -2 \lambda^{2} e^{-u-v} & 0
\end{array}\right)
$$

where

$$
f_{1}=-u_{2}-\frac{1}{2} v_{2}+u_{1} v_{1}+\frac{1}{4}\left(u_{1}^{2}+v_{1}^{2}\right), \quad f_{2}=u_{2}-\frac{1}{2} v_{2}-u_{1} v_{1}+\frac{1}{4}\left(u_{1}^{2}+v_{1}^{2}\right) .
$$

The matrices $U_{2}$ and $V_{2}$ realize the zero curvature representation of system (3.9).

B. Substitution $(3.1) \rightarrow(3.17)$ reduces matrix $U$ from (5.11) to the following form

$$
\hat{U}=\left(\begin{array}{cccc}
0 & 1 & 0 & 0 \\
(k-v) / 3+R & 0 & \lambda & 0 \\
0 & 0 & 0 & 1 \\
\lambda & 0 & (k-v) / 3-R & 0
\end{array}\right),
$$

where $R=\sqrt{2\left(u_{1}+v^{2}\right)} / 6$. It is obvious that one cannot remove $u_{1}$ from $\hat{U}$ by a gauge transformation. It is clear from the structure of the matrix $\hat{U}$ that

$$
\begin{aligned}
U_{t}- & V_{x}+[U, V]=A\left(k v_{1}+\frac{1}{2} u_{2}-\frac{1}{2} v_{3}-v_{t}\right) \\
& +B D_{x}\left(u_{3}-\frac{3}{4} \frac{\left(2 v v_{1}+u_{2}\right)^{2}}{v^{2}+u_{1}}+3 v v_{2}+\frac{3}{2} v_{1}^{2}+\frac{2}{3} v^{3}-k\left(2 v^{2}+u_{1}\right)-u_{t}\right),
\end{aligned}
$$


where $A$ and $B$ are some linearly independent matrices. Thus, this zero curvature representation for system (3.17) is generalized. Of course, one may introduce here the new variable $u^{\prime}=u_{1}$ to obtain an ordinary zero curvature representation. But we do not know if it is always possible.

C. Performing the substitution $(3.3) \rightarrow(3.6)$ into the matrix $U$ from (5.12) one obtains

$$
\hat{\tilde{U}}=\left(\begin{array}{cccc}
0 & 1 & 0 & 0 \\
-u / 3-\lambda & 0 & 1 & 0 \\
0 & 0 & 0 & 1 \\
u_{2} / 6+\left(u^{2}+v\right) / 9 & 0 & \lambda-u / 3 & 0
\end{array}\right) .
$$

The first gauge transformation is performed using $S_{1}=\exp \left(u_{1}\left(\partial \hat{\tilde{U}} / \partial u_{2}\right)\right)$ :

$$
S_{1}=\left(\begin{array}{cccc}
1 & 0 & 0 & 0 \\
0 & 1 & 0 & 0 \\
0 & 0 & 1 & 0 \\
u_{1} / 6 & 0 & 0 & 1
\end{array}\right)
$$

The transformed $U$-matrix is

$$
\tilde{U}_{1}=\left(\begin{array}{cccc}
0 & 1 & 0 & 0 \\
-u / 3-\lambda & 0 & 1 & 0 \\
u_{1} / 6 & 0 & 0 & 1 \\
\left(u^{2}+v\right) / 9 & -u_{1} / 6 & \lambda-u / 3 & 0
\end{array}\right) .
$$

The second gauge transformation is performed using $S_{2}=\exp \left(u\left(\partial \tilde{U}_{1} / \partial u_{1}\right)\right)$ :

$$
S_{2}=\left(\begin{array}{cccc}
1 & 0 & 0 & 0 \\
0 & 1 & 0 & 0 \\
u / 6 & 0 & 1 & 0 \\
0 & -u / 6 & 0 & 1
\end{array}\right)
$$

The result of the twofold gauge transformation is

$$
\begin{aligned}
\tilde{U}_{2} & =\left(\begin{array}{cccc}
0 & 1 & 0 & 0 \\
-u / 6-\lambda & 0 & 1 & 0 \\
0 & -u / 3 & 0 & 1 \\
u^{2} / 36+v / 9 & 0 & \lambda-u / 6 & 0
\end{array}\right), \\
\tilde{V}_{2} & =\left(\begin{array}{cccc}
-u_{1} / 6 & 2 \lambda & 0 & -2 \\
f_{3}-\lambda u / 3 & -u_{1} / 6 & u / 3 & 0 \\
\left(u u_{1}-v_{1}\right) / 18-\lambda u_{1} / 3 & -u_{2} / 3-u^{2} / 6-2 v / 9 & u_{1} / 6 & -2 \lambda \\
f_{4} & \left(v_{1}-u u_{1}\right) / 18-\lambda u_{1} / 3 & f_{3}+\lambda u / 3 & u_{1} / 6
\end{array}\right),
\end{aligned}
$$

where

$$
f_{3}=-\frac{1}{6} u_{2}-\frac{1}{18} u^{2}-\frac{2}{9} v-2 \lambda^{2}, \quad f_{4}=\frac{1}{18}\left(u u_{2}-v_{2}+u_{1}^{2}\right)+\frac{1}{108} u^{3}-\frac{1}{27} u v-\frac{2}{3} u \lambda^{2} .
$$

Matrices $\tilde{U}_{2}$ and $\tilde{V}_{2}$ realize the zero curvature representation of system (3.6).

\section{Conclusion}

The examples in Section 5 illustrate the fact that some systems possess ordinary zero curvature representation while others possess generalized zero curvature representation. All these 
representations are obtained from the Drinfeld-Sokolov $L, A$ operators by using corresponding differential substitutions listed in Section 4. Matrices $U$ and $V$ that realize all zero curvature representations have the size $4 \times 4$. Thus, the two-field evolutionary systems presented above are integrable in principle by the inverse spectral transform method. But the fact is that the inverse scattering problem for differential equations with order more than two is extremely difficult. That is why other methods for solution of equations may be useful [40]. They may be Bäcklund transformations [41], Darboux transformations [42, 43], Hirota method [44] or numeric simulating (see [45], for example).

\section{Acknowledgments}

We are grateful to Professor V.V. Sokolov for helpful discussions. This work was supported by Federal Agency for Education of Russian Federation, project \# 1.5.07.

\section{References}

[1] Ablowitz M.J., Segur M., Solitons and the inverse scattering transform, SIAM, Philadelphia, 1981.

[2] Zakharov V.E., Manakov S.V., Novikov S.P., Pitaevsky L.P., Theory of solitons: inverse problem method, Nauka, Moscow, 1980.

[3] Drinfeld V.G., Sokolov V.V., New evolution equations having (L-A) pairs, Trudy Sem. S.L. Soboleva, Inst. Mat., Novosibirsk 2 (1981), 5-9 (in Russian).

[4] Hirota R., Satsuma J., Soliton solutions of a coupled Korteweg-de Vries equation, Phys. Lett. A 85 (1981), 407-408.

[5] Antonowicz M., Fordy A., Coupled KdV equations with multi-Hamiltonian structures, Phys. D 28 (1987), $345-357$.

[6] Ma W.-X. A class of coupled KdV systems and their bi-Hamiltonian formulation, J. Phys. A: Math. Gen. 31 (1998), 7585-7591, solv-int/9803009.

[7] Ma W.-X., Pavlov M., Extending Hamiltonian operators to get bi-Hamiltonian of coupled KdV systems, Phys. Lett. A 246 (1998), 511-522, solv-int/9807002.

[8] Fuchssteiner B., The Lie algebra structure of degenerate Hamiltonian and bi-Hamiltonian systems, Progr. Theoret. Phys. 68 (1982), 1082-1104.

[9] Nutku Y., Oğuz Ö., Bi-Hamiltonian structure of a pair of coupled KdV equations, Nuovo Cimento 105 (1990), 1381-1383.

[10] Gerdt V.P., Zharkov A.Y., Computer classification of integrable coupled KdV-like systems, J. Symbolic Comput. 10 (1990), 203-207.

[11] Foursov M.V., On integrable coupled KdV-type systems, Inverse Problems 16 (2000), 259-274.

[12] Zharkov A.Y., Computer classification of the integrable coupled KdV-like systems with unit main matrix, J. Symbolic Comput. 15 (1993), 85-90.

[13] Karasu A., Painlevé classification of coupled Korteweg-de Vries-type systems, J. Math. Phys. 38 (1997), 3616-3622.

[14] Foursov M.V., Towards the complete classification of homogeneous two-component integrable equations, J. Math. Phys. 44 (2003), 3088-3096.

[15] Meshkov A.G., Sokolov V.V., Integrable evolution equations on the $N$-dimensional sphere, Comm. Math. Phys. 232 (2002), 1-18.

[16] Meshkov A.G., Sokolov V.V., Classification of integrable divergent $N$-component evolution systems, Theoret. and Math. Phys. 139 (2004), 609-622.

[17] Balakhnev M.Ju., A class of integrable evolutionary vector equations. Theoret. and Math. Phys. 142 (2005), 8-14.

[18] Balakhnev M.Ju., Meshkov A.G., Integrable anisotropic evolution equations on a sphere, SIGMA 1 (2005), 027, 11 pages, nlin.SI/0512032.

[19] Balakhnev M.Ju., Meshkov A.G., On a classification of integrable vectorial evolutionary equations, submitted. 
[20] Kulemin I.V., Meshkov A.G., To the classification of integrable systems in 1+1 dimensions, in Proceedings Second International Conference "Symmetry in Nonlinear Mathematical Physics" (July 7-13, 1997, Kyiv), Editors M. Shkil, A. Nikitin and V. Boyko, Institute of Mathematics, Kyiv, 1997, 115-121.

[21] Meshkov A.G., On symmetry classification classification of third order evolutionary systems of divergent type, Fundam. Prikl. Mat. 12 (2006), no. 7, 141-161 (in Russian).

[22] Ibragimov N.Kh., Shabat A.B., On infinite Lie-Bäcklund algebras, Funktsional. Anal. i Prilozhen. 14 (1980), no. 4, 79-80 (in Russian).

[23] Svinolupov S.I., Sokolov V.V., Evolution equations with nontrivial conservation laws, Funktsional. Anal. $i$ Prilozhen. 16 (1982), no. 4, 86-87.

[24] Sokolov V.V., Shabat A.B., Classification of integrable evolution equations, Soviet Scientific Reviews, Section C 4 (1984), 221-280.

[25] Mikhailov A.V., Shabat A.B., Yamilov R.I., The symmetry approach to the classification of integrable equations. Complete lists of integrable systems, Russian Math. Surveys 42 (1987), no. 4, 1-63.

[26] Mikhailov A.V., Shabat A.B., Sokolov V.V., The symmetry approach to the classification of integrable equations, in Integrability and Kinetic Equations for Solitons, Editors V.G. Bahtariar, V.E. Zakharov and V.M. Chernousenko, Naukova Dumka, Kyiv, 1990, 213-279 (English transl.: in What is Integrability?, Springer-Verlag, New York, 1991, 115-184).

[27] Fokas A.S., Symmetries and integrability, Stud. Appl. Math. 77 (1987), 253-299.

[28] Galindo A., Martinez L., Kernels and ranges in the variational formalism, Lett. Math. Phys. 2 (1978), 385-390.

[29] Drinfeld V.G., Sokolov V.V., Lie algebras and equations of Korteweg-de Vries type, Current Problems in Mathematics, Vol. 24, Itogi Nauki i Tekhniki, VINITI, Moscow, 1984, 81-180 (English transl.: J. Sov. Math. 30 (1985), 1975-2035).

[30] Balakhnev M.Yu., Kulemin I.V., Differential substitutions for third-order evolution systems, Differ. Uravn. Protsessy Upr. (2002), no. 1, 7 pages (in Russian), available at http://www.neva.ru/journal/j/.

[31] Zakharov V.E., Shabat A.B., Exact theory of two-dimensional self-focusing and one-dimensional selfmodulation of waves in nonlinear media, Ž. Eksper. Teoret. Fiz. 61 (1971), no. 1, 118-134 (English transl.: Soviet Physics JETP 34 (1972), no. 1, 62-69).

[32] Chen H.H., Lee Y.C., Liu C.S., Integrability of nonlinear Hamiltonian systems by inverse scattering method, Phys. Scripta 20 (1979), 490-492.

[33] Meshkov A.G., Necessary conditions of the integrability, Inverse Problems 10 (1994), 635-653.

[34] Fuchssteiner B., Fokas A.S., Symplectic structures, their Bäcklund transformations and hereditary symmetries, Phys. D 4 (1981/82), 47-66.

[35] Drinfeld V.G., Sokolov V.V., Equations that are related to the Korteweg-de Vries equation, Dokl. Akad. Nauk SSSR 284 (1985), no. 1, 29-33 (in Russian).

[36] Meshkov A.G., Tools for symmetry analysis of PDEs, Differ. Uravn. Protsessy Upr. (2002), no. 1, 53 pages, available at http://www.neva.ru/journal/j/.

[37] Lax P.D., Integrals of nonlinear equations of evolution and solitary waves, Comm. Pure Appl. Math. 21 (1968), 467-490.

[38] Manakov S.V., The method of the inverse scattering problem, and two-dimensional evolution equations, Uspehi Mat. Nauk 31 (1976), no. 5, 245-246 (in Russian).

[39] Zakharov V.E., Inverse scattering method, in Solitons, Editors R.K. Bullough and P.J. Caudrey, SpringerVerlag, New York, 1980, 270-309.

[40] Dodd R.K., Eilbeck J.C., Gibbon J.D., Morris H.C., Solitons and nonlinear wave equations, Academic Press Inc., London, 1984.

[41] Miura R.M. (Editors), Bäcklund transformations, the inverse scattering method, solitons, and their applications, NSF Research Workshop on Contact Transformations, Lecture Notes in Mathematics, Vol. 515, 1976.

[42] Matveev V.B., Salle M.A., Darboux transformations and solitons, Springer, Berlin, 1991.

[43] Gu C.H., Hu H.S., Zhou Z.X., Darboux transformations in soliton theory and its geometric applications, Shanghai Scientific and Technical Publishers, Shanghai, 1999.

[44] Hirota R., The direct method in soliton theory, Cambridge University Press, Cambridge, 2004.

[45] Zienkiewcz O.C., Morgan K., Finite elements and approximation, Wiley-Interscience Publication, New York, 1983. 